Mathematical Medicine and Biology (2014) 31, 259-283

doi:10.1093/imammb/dqt009

Advance Access publication on June 10, 2013

\title{
Mathematical modelling of active contraction in isolated cardiomyocytes
}

\author{
RICARDO RUIZ-BAIER* \\ CMCS-MATHICSE-SB, Ecole Polytechnique Fédérale de Lausanne, CH-1015 Lausanne, Switzerland \\ *Corresponding author: ricardo.ruiz@epfl.ch
}

Alessio GizzI

Nonlinear Physics and Mathematical Modeling Laboratory, University Campus Bio-Medico of Rome, I-00128 Rome, Italy

Simone Rossi

CMCS-MATHICSE-SB, Ecole Polytechnique Fédérale de Lausanne, CH-1015 Lausanne, Switzerland

Christian Cherubini

Nonlinear Physics and Mathematical Modeling Laboratory, University Campus Bio-Medico of Rome, I-00128 Rome, Italy

AYMEn LAADHARI

CMCS-MATHICSE-SB, Ecole Polytechnique Fédérale de Lausanne, CH-1015 Lausanne, Switzerland

SimonetTa FILIPPI

Nonlinear Physics and Mathematical Modeling Laboratory, University Campus Bio-Medico of Rome, I-00128 Rome, Italy

AND

\section{ALFIO QUARTERONI}

CMCS-MATHICSE-SB, Ecole Polytechnique Fédérale de Lausanne, CH-1015 Lausanne, Switzerland MOX - Politecnico de Milano, piazza Leonardo da Vinci 32, 20133 Milano, Italy

[Received on 22 October 2012; revised on 5 March 2013; accepted on 14 May 2013]

We investigate the interaction of intracellular calcium spatio-temporal variations with the self-sustained contractions in cardiac myocytes. A consistent mathematical model is presented considering a hyperelastic description of the passive mechanical properties of the cell, combined with an active-strain framework to explain the active shortening of myocytes and its coupling with cytosolic and sarcoplasmic calcium dynamics. A finite element method based on a Taylor-Hood discretization is employed to approximate the nonlinear elasticity equations, whereas the calcium concentration and mechanical activation variables are discretized by piecewise linear finite elements. Several numerical tests illustrate the ability of the model in predicting key experimentally established characteristics including: (i) calcium propagation patterns and contractility, (ii) the influence of boundary conditions and cell shape on the onset of structural and active anisotropy and (iii) the high localized stress distributions at the focal adhesions. Besides, they also highlight the potential of the method in elucidating some important subcellular mechanisms affecting, e.g. cardiac repolarization.

Keywords: cardiomyocyte modelling; active-strain contraction; nonlinear elasticity; calcium propagation; finite element formulation; coupled multiphysics. 


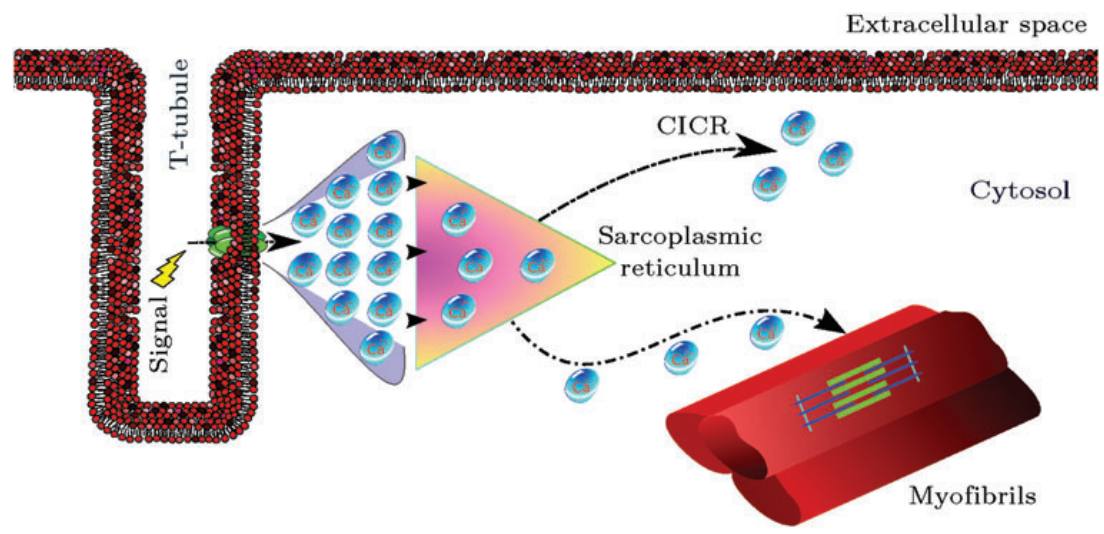

FIG. 1. Schematic representation of the functional components of an individual cardiomyocyte. Influx of $\mathrm{Ca}^{2+}$ into the cell increases intracellular $\left[\mathrm{Ca}^{2+}\right]$, inducing a release of $\mathrm{Ca}^{2+}$ from the sarcoplasmic reticulum towards cytosol and activating the myofilaments.

\section{Introduction}

Although it has been widely accepted, since several years, that numerous cell types respond to mechanical stimuli, no unanimous agreement can be found as to which specific stimuli cells directly respond: stress, strain, strain-rate, strain energy, etc. (Humphrey, 2001). From a physical point of view, it is well established that stress and strain concepts are fundamental in order to gain essential information necessary in building up phenomenological constitutive models in mechanobiology. Such a procedure is rapidly becoming a standard approach towards both the basic understanding of the biological system properties and the design of experiments and medical applications. From a biomechanical point of view, therefore, cardiovascular functions and development need the partnership of several different scientific fields (Taber, 2001), i.e. biology, physics, mathematics, engineering, statistics and medicine.

In the last decade, several examples of different muscle organizations have been presented on an experimental basis (Parker et al., 2008; Goldmann, 2012; Marshall \& Lumpkin, 2012). The leading ideas consider muscular organization as the product of a full functional adaptation of the cell spanning from the sarcomere to the muscle bundle (Sheehy et al., 2012). Through such an approach boundary constraints in the extracellular space have been recognized to potentiate the organization of cytoskeletal scaffolds for sarcomerogenesis, generating a positive feedback loop which polarizes the contractile cytoskeleton towards the functional organization of the tissue (Grosberg et al., 2011). Moreover, cell-cell and cell-matrix adhesions have been suggested to cooperate for the cardiac function as an electromechanical syncytium (McCain et al., 2012).

The excitation-contraction mechanisms in the cardiac muscle are coordinated by an autonomous electrical activation generated in the sino-atrial node and propagated through the heart wall (Keener \& Sneyd, 1998). The cardiomyocyte is composed of myofibrils bundles containing sarcomeres, the cell contractile units, consisting of thick and thin myofilaments, myosin and actin proteins. At the microscale (see Fig. 1), the exchange of calcium between cytosol and the sarcoplasmic reticulum influences the interaction of these myofilaments which onsets the shortening of the sarcomeres and drives the process of excitation-contraction of the whole cardiac cell. During excitation the depolarization of the sarcolemma induces the influx of extracellular calcium into the cardiomyocytes; the increase of intracellular calcium induces more $\mathrm{Ca}^{2+}$ to be released from the sarcoplasmic reticulum; cytosolic $\mathrm{Ca}^{2+}$ ions 
bind to troponin-C and activate the myofilaments. Although the excitation-contraction mechanism and the specific process of Calcium-induced calcium release (CICR) taking place at the sub-cellular level have major evidences both at the theoretical (Stern, 1992) and experimental (Fabiato, 1989) levels, the full understanding of the exact interplay between the different processes is still lacking. These processes play a key role in the overall cardiac function, and therefore understanding of these mechanisms can be of utmost importance and interest for the study of many physiological and pathological heart conditions (Hatano et al., 2011; Bers, 2002).

Several systemic effects of cardiac mechano-electric interactions can be studied by regarding elastic properties of isolated cardiac myocytes (Iribe et al., 2009; Grosberg et al., 2011; McCain et al., 2012; Sheehy et al., 2012). Experimental evidences (Deshpande et al., 2006) highlight that the largest forces recorded are often present at locations where no visible stress fibres exist. Therefore stresses are induced by micro-structure remodelling acting on a much finer scale. This important point justifies the choice of a contractility model formulated at the continuum level, capable of characterizing such phenomena rather than the description of an ensemble of discrete particles. These homogenization and generalization procedures are based on the following key assumptions: (i) there is sufficient calcium in the cell so that the mechanical activation is not limited by its availability; (ii) a representative volume element, much smaller than the dimensions of the cell, can be identified; and (iii) the mechanical response is observable from any direction.

In particular, we provide a quantitative simulation of the behaviour of single cells under a precise set of experimental conditions by proposing a novel chemo-active approach. Ionic kinetics and voltage-dependent equations describing potential generation and propagation at the cellular level are important ingredients in cardiac electrophysiology (Pullan et al., 2005). However, in this preliminary study, we focus on describing quantitatively the behaviour of the principal calcium quantities inside the cardiomyocyte, noting that physiological models for $\left[\mathrm{Ca}^{2+}\right]$ dynamics are able to mimic the coupling of $\mathrm{Ca}^{2+}$ with voltage (Bers, 2002).

Our mathematical model is based on an active-strain formulation for the description of the cardiomyocyte active mechanical response following Cherubini et al. (2008) and Ruiz-Baier et al. (2013). In this approach, the mechanical activation may be represented as a virtual multiplicative splitting of the deformation gradient into a passive elastic response, and an active deformation depending directly on the nonlinear dynamics that describe chemical reactions between calcium species, CICR. Although the adopted calcium model is a well-established simplified version of the more complex ionic dynamics of the cell physiology (Goldbeter et al., 1990; Sneyd et al., 2005; Tracqui et al., 2008), we are able to quantitatively represent, at the same time, both the anisotropic passive intracellular organization, i.e. the T-tubule system, and the anisotropic active emerging cellular structures, that may be important in the single myocyte deformation and calcium regulation.

Inspired by Stålhand et al. (2008), we derive a model that is thermodynamically consistent in the sense that the second law of thermodynamics is satisfied. Such a generalized model must be able to characterize interactions among the ionic quantities, the cellular mechanical properties and the environmental effects. Moreover, it must explain the influence of cell shape and boundary conditions on the onset of structural anisotropies and stress concentrations. We demonstrate that the present model is capable of addressing all of these requirements. With the aid of finite-element-based simulations we will address the feasibility and adequateness of employing a macroscopic description of the mechanochemical behaviour of a single cell adopting an active strain approach. Such a modelling strategy can therefore be used to explain the relation between microscopic cell dynamics and macroscopic cardiac function. Model validation is performed in terms of calcium wave propagation velocity and active force vs. sarcomere length relationship. 
The outline of the present work is as follows. In Section 2, we introduce the notation and the main elements of the mechano-chemical model, namely, the elastic properties of the single cell, its activation and the mechanisms driving the propagation of intracellular calcium. Section 3 is devoted to the presentation of our numerical method and to the discussion of the application to cardiomyocyte selfsustained contractions. We also address some numerical experiments validating models and methods. Some conclusions and discussion about limitations and potential extensions of this work are provided in Section 4. Finally, details on the linearized momentum equations and algorithmic description are addressed in the Appendix.

\section{Coupled model based on an active-strain description}

\subsection{Kinematics}

Let $\mathbf{x}$ represent the current position of a material particle of the myocyte $\Omega_{t}$ that occupied the position $\mathbf{X}$ in the reference configuration $\Omega \subset \mathbb{R}^{d}, d=2,3$. By $\Gamma$ we denote the smooth boundary of $\Omega$ decomposed into a stress-free and a fixed boundary, $\Gamma_{\mathrm{N}}$ and $\Gamma_{\mathrm{D}}$, respectively (defined on the reference domain). From the deformation gradient tensor $\mathbf{F}=\nabla \mathbf{x}$, we derive the right and left Cauchy-Green deformation tensors, $\mathbf{C}=\mathbf{F}^{\mathrm{T}} \mathbf{F}$ and $\mathbf{B}=\mathbf{F F}^{\mathrm{T}}$, respectively, and the Jacobian $J=\operatorname{det} \mathbf{F}$ characterizing the tangential volume map. Here $\nabla=\left(\partial / \partial \mathbf{X}_{k} \mathbf{e}_{k}\right)$ stands for the material gradient.

The properties of hyperelastic materials are characterized by scalar stored energy functions whose derivatives with respect to strain give stress measures. We suppose that the passive material response of the isolated cell can be described through an incompressible transversely isotropic constitutive law. The strain energy function reads (see, e.g. Holzapfel, 2000; Holzapfel \& Ogden, 2009)

$$
\mathcal{W}(\mathbf{F})=\frac{a}{2 b} \exp \left(b\left[I_{1}-d\right]\right)+\frac{a_{f}}{2 b_{f}}\left[\exp \left(b_{f}\left[I_{4, f}-1\right]^{2}\right)-1\right]-p(J-1),
$$

where $p$ is a Lagrange multiplier to impose incompressibility, usually identified as the pressure field, $a$ is a shear modulus (a summary of the required parameter values and units is provided in Table 1), $d$ is the spatial dimension, $a_{f}, b, b_{f}$ are experimentally fitted, and the invariants $I_{i}$ are defined as $I_{1}:=\operatorname{tr}(\mathbf{C})$, $I_{4, f}:=\mathbf{F f}_{0} \cdot \mathbf{F f}_{0}$, where $\mathbf{f}_{0}$ is a unitary direction vector in the reference configuration representing the myofibrils direction and is the scalar product of two vectors. The first Piola-Kirchhoff stress tensor then reads

$$
\mathbf{P}=\frac{\partial \mathcal{W}}{\partial \mathbf{F}}=a \exp \left(b\left[I_{1}-d\right]\right) \mathbf{F}+2 a_{f}\left(I_{4, f}-1\right) \exp \left(b_{f}\left[I_{4, f}-1\right]^{2}\right) \mathbf{F} \mathbf{f}_{0} \otimes \mathbf{f}_{0}-p J \mathbf{F}^{-\mathrm{T}} .
$$

In order to account for the activation of the cardiac contraction, we follow a model of active strain (Nardinocchi \& Teresi, 2007; Cherubini et al., 2009; Rossi et al., 2012), where we assume a multiplicative decomposition of the deformation gradient into a passive and an active component, $\mathbf{F}=\mathbf{F}_{\mathrm{E}} \mathbf{F}_{\mathrm{A}}$. This assumption means that the energy is not stored as strain energy, rather it corresponds to an internal rearrangement of the material that does not produce macroscopic deformation. This fictitious process is represented by an elastic intermediate configuration between the reference and the current configurations (Fig. 2). The active deformation gradient assumes the generic form

$$
\mathbf{F}_{\mathrm{A}}=\mathbf{I}+\gamma_{\mathrm{f}} \mathbf{f}_{0} \otimes \mathbf{f}_{0}+\gamma_{\mathrm{s}} \mathbf{s}_{0} \otimes \mathbf{s}_{0}+\gamma_{\mathrm{n}} \mathbf{n}_{0} \otimes \mathbf{n}_{0},
$$

where $\gamma_{\mathrm{f}}, \gamma_{\mathrm{s}}$ and $\gamma_{\mathrm{n}}$ are smooth scalar activation functions and $\otimes$ stands for the usual tensor product of two vectors. For convenience we introduce an orthogonal frame of reference $\left(\mathbf{f}_{0}, \mathbf{s}_{0}, \mathbf{n}_{0}\right)$, where the 
TABLE 1 Values and units for the material and CICR parameters

\begin{tabular}{lcccccccccccc}
\hline$\alpha$ & $a$ & $v_{1}$ & $v_{2}$ & $v_{3}$ & $v_{4}$ & $v_{5}$ & $k_{2}$ & $k_{3}$ & $k_{4}$ & $D_{f}$ & $D_{s}$ & $D_{n}$ \\
\hline 0.01 & 0.496 & 1.58 & 16 & 91 & 2 & 0.2 & 1 & 4 & 0.7481 & 60 & 30 & 30 \\
$(\mathrm{kPa})$ & $(\mathrm{kPa})$ & $\left(\mu \mathrm{Ms}^{-1}\right)$ & $\left(\mu \mathrm{Ms}^{-1}\right)$ & $\left(\mu \mathrm{Ms}^{-1}\right)$ & $\left(\mathrm{s}^{-1}\right)$ & $\left(\mathrm{s}^{-1}\right)$ & $(\mu \mathrm{M})$ & $(\mu \mathrm{M})^{2}$ & $(\mu \mathrm{M})^{4}$ & $\left(\mu \mathrm{m}^{2} \mathrm{~s}^{-1}\right)$ & $\left(\mu \mathrm{m}^{2} \mathrm{~s}^{-1}\right)$ & $\left(\mu \mathrm{m}^{2} \mathrm{~s}^{-1}\right)$ \\
\hline
\end{tabular}

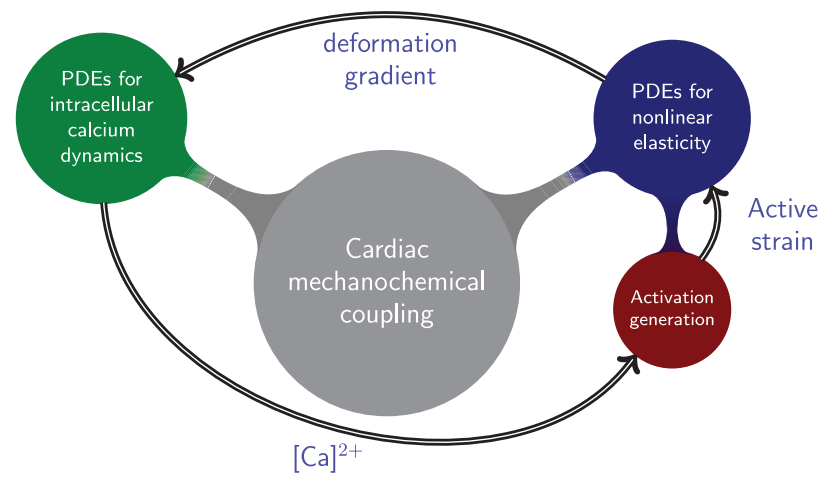

FIG. 2. Sketch of the multiphysics coupling strategy.

directions $\mathbf{s}_{0}$ and $\mathbf{n}_{0}$ can be chosen arbitrarily, orthogonal to $\mathbf{f}_{0}$, and we also denote any of these directions by the generic vector $\mathbf{l}_{0} \in\left\{\mathbf{f}_{0}, \mathbf{s}_{0}, \mathbf{n}_{0}\right\}$. In this manner $\mathbf{F}_{\mathrm{A}}$ describes the active deformations of the cell in the three principal directions. We can accordingly define the following quantities for the intermediate motion: $\mathbf{C}_{\mathrm{A}}=\mathbf{F}_{\mathrm{A}}^{\mathrm{T}} \mathbf{F}_{\mathrm{A}}, \mathbf{F}_{\mathrm{E}}=\mathbf{F} \mathbf{F}_{\mathrm{A}}^{-1}, \mathbf{C}_{\mathrm{E}}=\mathbf{F}_{\mathrm{A}}^{-\mathrm{T}} \mathbf{C} \mathbf{F}_{\mathrm{A}}^{-1}$, and $J_{\mathrm{A}}=\operatorname{det} \mathbf{F}_{\mathrm{A}}, J_{\mathrm{E}}=\operatorname{det} \mathbf{F}_{\mathrm{E}}, J=J_{\mathrm{E}} J_{\mathrm{A}}$. In the aforementioned new configuration, the strain energy function is rewritten in terms of $\mathbf{F}_{\mathrm{E}}$, and then pulled back to the reference configuration. This new stored energy can now be written as a function of the following modified invariants:

$$
\begin{aligned}
& I_{1}^{\mathrm{E}}:=\operatorname{tr}\left(\mathbf{C}_{\mathrm{E}}\right)=\operatorname{tr}\left(\mathbf{F}^{\mathrm{T}} \mathbf{C}_{\mathrm{A}}^{-1} \mathbf{F}\right)=I_{1}-\sum_{l \in\{f, s, n\}} \frac{\gamma_{1}\left(\gamma_{1}+2\right)}{\left(\gamma_{1}+1\right)^{2}} \mathbf{F l}_{0} \cdot \mathbf{F} \mathbf{l}_{0}, \\
& I_{4, f}^{\mathrm{E}}:=\mathbf{F}_{\mathrm{E}} \mathbf{f}_{0} \cdot \mathbf{F}_{\mathrm{E}} \mathbf{f}_{0}=\mathbf{F F}_{\mathrm{A}}^{-1} \mathbf{f}_{0} \cdot \mathbf{F} \mathbf{F}_{\mathrm{A}}^{-1} \mathbf{f}_{0}=\left(1-\frac{\gamma_{\mathrm{f}}}{1+\gamma_{\mathrm{f}}}\right)^{2} \mathbf{F f}_{0} \cdot \mathbf{F} \mathbf{f}_{0}=\left(1+\gamma_{\mathrm{f}}\right)^{-2} I_{4, f} .
\end{aligned}
$$

The field $\gamma_{\mathrm{f}}$ represents the active shortening of the cardiomyocytes, whereas $\gamma_{\mathrm{s}}, \gamma_{\mathrm{n}}$ will take into account the corresponding thickening, in order to satisfy the incompressibility of the cell itself (Iribe et al., 2007), incorporated through the condition $J_{\mathrm{A}}=1$. Such an assumption also permits $\gamma_{\mathrm{s}}$ and $\gamma_{\mathrm{n}}$ to be defined in terms of $\gamma_{\mathrm{f}}$. In two dimensions we set $\gamma_{\mathrm{n}}=0$, such that $\gamma_{\mathrm{s}}=\left(\gamma_{\mathrm{f}}+1\right)^{-1}-1$, while for $d=3$ we put $\gamma_{\mathrm{n}}=\gamma_{\mathrm{s}}=\left(\gamma_{\mathrm{f}}+1\right)^{-1 / 2}-1$. The (activated) Piola-Kirchhoff stress tensor assumes the following expression:

$$
\mathbf{P}=2 \psi_{1}^{\mathrm{E}}\left[\left(1+\gamma_{\mathrm{f}}\right)^{2} \mathbf{F}-g\left(\gamma_{\mathrm{f}}\right) \mathbf{F} \mathbf{f}_{0} \otimes \mathbf{f}_{0}\right]+\frac{2}{\left(1+\gamma_{\mathrm{f}}\right)^{2}} \psi_{4, f}^{\mathrm{E}} \mathbf{F} \mathbf{f}_{0} \otimes \mathbf{f}_{0}-p J \mathbf{F}^{\mathrm{T}}
$$


where $g\left(\gamma_{\mathrm{f}}\right)=1 /\left(1+\gamma_{\mathrm{f}}\right)^{2}-\left(1+\gamma_{\mathrm{f}}\right)^{2} \quad$ and $\quad \psi_{1}^{\mathrm{E}}=(a / 2) \exp \left(b\left[I_{1}^{\mathrm{E}}-3\right]\right), \quad \psi_{4, f}^{\mathrm{E}}=a_{f}\left(I_{4, f}^{\mathrm{E}}-1\right) \exp \left(b_{f}\right.$ $\left[I_{4, f}^{\mathrm{E}}-1\right]^{2}$ ) (details on the derivation can be found in Rossi et al., 2012). Note that the second term of the RHS in (2.1) represents an extra stress associated with an elastic deformation of the cross-bridges (Stålhand et al., 2011). The precise constitutive form of the activation will be postponed to Section 2.3.

\subsection{Calcium dynamics}

The mechanisms of calcium wave propagation show diffusion of $\left[\mathrm{Ca}^{2+}\right]$, coupled to CICR, from channels sensitive to ryanodine release in the sarcoplasmic reticulum (Subramanian et al., 2001). Digital imaging techniques and intracellular calcium indicators provide insights concerning calcium distribution inside myocytes (Böl et al., 2012). Here, the interaction between cytosolic and sarcoplasmic calcium concentrations will be modelled following Tracqui et al. (2008) and Goldbeter et al. (1990). The system of partial differential equations governing the simplified nonlinear dynamics of the CICR behaviour consists of the mass balance equations

$$
\begin{aligned}
& \partial_{t} w_{\mathrm{c}}=\operatorname{div}\left(\mathbf{D}(\mathbf{F}) \nabla w_{\mathrm{c}}\right)+K\left(w_{\mathrm{c}}, w_{\mathrm{s}}\right), \\
& \partial_{t} w_{\mathrm{s}}=L\left(w_{\mathrm{c}}, w_{\mathrm{s}}\right) \quad \text { in } \Omega \times(0, T),
\end{aligned}
$$

where $w_{\mathrm{c}}$ and $w_{\mathrm{s}}$ represent the concentrations of cytosolic and sarcoplasmic calcium, respectively. Only two calcium species are considered under the assumption that the level of $I P_{3}$ remains constant during external stimulation (Goldbeter et al., 1990). The anisotropy of the calcium propagation (Subramanian et al., 2001) along with the effect of the domain motion are modelled by the tensor $\mathbf{D}=\mathbf{F}^{-1} \operatorname{diag}\left(D_{f}, D_{s}, D_{n}\right) \mathbf{F}^{-\mathrm{T}}$, where $D_{f}, D_{s}, D_{n}$ are diffusivities of cytosolic calcium in three orthogonal directions. This expression is obtained from a pull-back of the diffusion in the deformed configuration. All differential operators involved are considered with respect to Lagrangian coordinates. The nonlinear reaction terms are given by

$$
\begin{aligned}
& K\left(w_{\mathrm{c}}, w_{\mathrm{s}}\right)=v_{1}-\frac{v_{2} w_{\mathrm{c}}^{2}}{k_{2}+w_{\mathrm{c}}^{2}}+\frac{v_{3} w_{\mathrm{c}}^{4} w_{\mathrm{s}}^{2}}{\left(k_{3}+w_{\mathrm{s}}^{2}\right)\left(k_{4}+w_{\mathrm{c}}^{4}\right)}-v_{4} w_{\mathrm{c}}, \\
& L\left(w_{\mathrm{c}}, w_{\mathrm{s}}\right)=\frac{v_{2} w_{\mathrm{c}}^{2}}{k_{2}+w_{\mathrm{c}}^{2}}-\frac{v_{3} w_{\mathrm{c}}^{4} w_{\mathrm{s}}^{2}}{\left(k_{3}+w_{\mathrm{s}}^{2}\right)\left(k_{4}+w_{\mathrm{c}}^{4}\right)}-v_{5} w_{\mathrm{s}} .
\end{aligned}
$$

The term $v_{1}$ represents an inflow flux plus intracellular calcium pulses originated from the asynchrony of calcium pools receptors (Goldbeter et al., 1990), $v_{2}$ and $v_{3}$ account for low and high levels of free cytosolic calcium flux pumped from the sarcoplasmic reticulum, and $v_{4}$ models an efflux of calcium out of the cell following a chemical exchange process (Tracqui \& Ohayon, 2009). In Fig. 3, we observe the periodic behaviour of the associated dynamical system

$$
\partial_{t} w_{\mathrm{c}}=K\left(w_{\mathrm{c}}, w_{\mathrm{s}}\right), \partial_{t} w_{\mathrm{s}}=L\left(w_{\mathrm{c}}, w_{\mathrm{s}}\right)
$$

where sustained oscillations tend to a limit cycle. Even if such a formulation represents a simplification of the complex intra-extracellular calcium dynamics, our choice is motivated by the more general framework we consider from the chemo-mechanical viewpoint. 


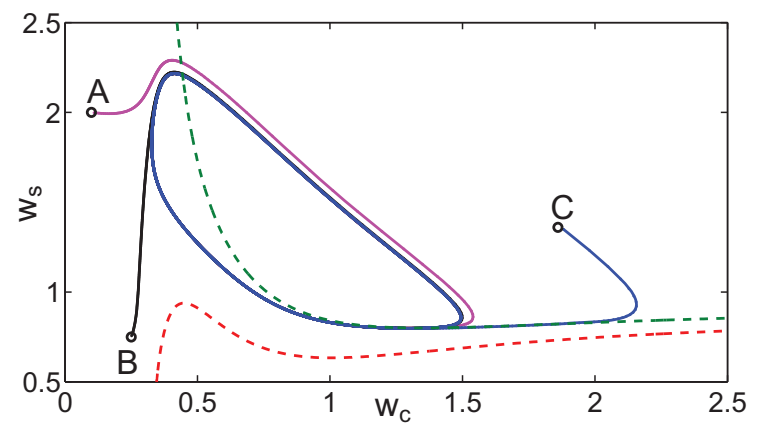

FIG. 3. Phase portrait for the dynamical system associated to (2.2). The system nullclines for $K=0$ and $L=0$ are the curves in green and red, respectively. Three computed trajectories after 2500 time steps are displayed starting from the states $A=(0.1,2)$, $B=(0.25,0.75)$ and $C=(1.85,1.35)$.

\subsection{Calcium-induced activation and thermodynamic considerations}

We derive a mathematical description of the activation mechanisms in the myofilaments using macroscopic arguments from thermodynamics, mainly motivated by analogous arguments widely used for the modelling of the overall cardiac function. A somewhat different macroscopic description, based on averaging the kinetics of the cross-bridges (as in the early model by Huxley, 1957) has been employed by Zahalak (1981). Another common strategy is based on a mean field approximation (see, e.g. Landesberg \& Sideman, 1994; Negroni \& Lascano, 2008; Katsnelson et al., 2009 and the references therein), where the complex behaviour of the myofilaments is described by a representative cross-bridge. Although this method is known to fail in reproducing the cooperative interactions in the sarcomeres (Rice \& de Tombe, 2004), promising results correlating well with Monte Carlo simulations of spatially detailed models are shown in Washio et al. (2012). More detailed descriptions for the cross-bridge kinetics could be independently incorporated in the present model without much effort. This aspect will be addressed in a forthcoming contribution. For the sake of simplicity, in our minimal model the activation is assumed to depend solely on the cytosolic calcium concentration, and the active shortening field $\gamma_{\mathrm{f}}$ obeys the differential equation

$$
\partial_{t} \gamma_{\mathrm{f}}=G\left(w_{\mathrm{c}}, \gamma_{\mathrm{f}}\right)
$$

representing the accumulation of a dimensional activation proportional to the exchange of cytosolic calcium concentration (Laadhari et al., 2012). As discussed in Stålhand et al. (2011), the internal power of the overall mechano-chemical system is

$$
\mathcal{P}=\mathbf{P}: \dot{\mathbf{F}}+\mathbf{P}_{\mathrm{A}}: \dot{\mathbf{F}}_{\mathrm{A}}+Q \dot{\mathbf{w}},
$$

where $\mathbf{P}_{\mathrm{A}}$ is the stress generated by the cell through the power stroke (see Stålhand et al., 2008), $\mathbf{w}=$ $\left(w_{\mathrm{c}}, w_{\mathrm{s}}\right)^{\mathrm{T}}$ is the vector of calcium concentrations and $Q$ is a chemical potential that drives the calcium currents. The internal total energy satisfies $\dot{\mathcal{E}}=\mathcal{P}+\mathcal{T}$, where $\mathcal{T}$ is the thermal power. The second law of thermodynamics then implies that $\dot{\eta} T-\mathcal{T} \geqslant 0$ (where $\eta$ is the entropy and $T$ stands for temperature), which, after introducing the free energy $\Upsilon=\Upsilon\left(\mathbf{F}, \mathbf{F}_{\mathrm{A}}, \mathbf{w}, T\right)=\mathcal{E}-\eta T$, rewrites as $\dot{\Upsilon} \leqslant \mathcal{P}-\eta \dot{T}$, and 


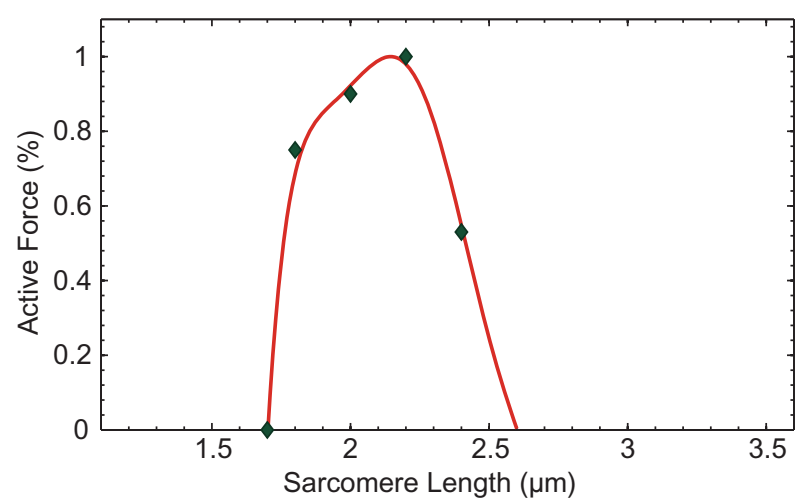

FIG. 4. Shape of the active force $P_{\mathrm{A}}$ given by (2.5) (solid line), and experimental results from Strobeck \& Sonnenblick (1986) (dots).

equivalently we arrive at

$$
\left(\mathbf{P}-\frac{\partial \Upsilon}{\partial \mathbf{F}}\right): \dot{\mathbf{F}}+\left(\mathbf{P}_{\mathrm{A}}-\frac{\partial \Upsilon}{\partial \mathbf{F}_{\mathrm{A}}}\right): \dot{\mathbf{F}}_{\mathrm{A}}+\left(Q-\frac{\partial \Upsilon}{\partial \mathbf{w}}\right) \cdot \dot{\mathbf{w}}-\left(\eta+\frac{\partial \Upsilon}{\partial T}\right) \dot{T} \geqslant 0
$$

Since the mechanical energy $\mathcal{W}$ is written in an intermediate configuration and then pulled back to the reference state, by assuming the vanishing of the first term in (2.4) we get the constitutive equation (2.1). In addition, we impose $Q=\partial \Upsilon / \partial \mathbf{w}$ and $\eta=-\partial \Upsilon / \partial T$, so that (2.4) holds whenever

$$
\mathbf{P}_{\mathrm{A}}-\frac{\partial \Upsilon}{\partial \mathbf{F}_{\mathrm{A}}}=\beta \dot{\mathbf{F}}_{\mathrm{A}}
$$

for some constant $\beta=\beta\left(\mathbf{w}, \mathbf{F}_{\mathrm{A}}\right) \geqslant 0$. Given the particular form of $\mathbf{F}_{\mathrm{A}}$, we can rewrite the free energy as a function of the minimal set of variables $\Upsilon=\Upsilon\left(\mathbf{F}, \gamma_{\mathrm{f}}, \mathbf{w}, T\right)$ and subsequently recast (2.5) in terms of $\gamma_{\mathrm{f}}$ and its time derivative as $P_{\mathrm{A}}-\partial \Upsilon / \partial \gamma_{\mathrm{f}}=\beta \dot{\gamma}_{\mathrm{f}}$, for $\beta=\beta\left(\mathbf{w}, \gamma_{\mathrm{f}}\right) \geqslant 0$. In this way, we provide a specification for the reaction term $G$ in (2.3) as follows:

$$
G\left(w_{\mathrm{c}}, \gamma_{\mathrm{f}}\right)=\beta^{-1}\left(P_{\mathrm{A}}-\left[2\left(1+\gamma_{\mathrm{f}}\right) I_{1}+g^{\prime}\left(\gamma_{\mathrm{f}}\right) I_{4, f}\right] \psi_{1}^{\mathrm{E}}-\frac{2}{\left(1+\gamma_{\mathrm{f}}\right)^{3}} I_{4, f} \psi_{4, f}^{\mathrm{E}}\right),
$$

where $g^{\prime}\left(\gamma_{\mathrm{f}}\right)=-2\left(1+\gamma_{\mathrm{f}}\right)-2 /\left(1+\gamma_{\mathrm{f}}\right)^{3}$. It only remains to assign a value to $\beta$ and to the thermodynamic force $P_{\mathrm{A}}$ driving the sarcomere contraction. We here propose to use $\beta=1 / 2 w_{\mathrm{c}}^{2} \geqslant 0$ and

$$
P_{\mathrm{A}}=\beta\left[\frac{d_{0}}{2}+\sum_{n=1}^{3} d_{n} \sin (n l)+e_{n} \cos (n l)\right],
$$

which depends directly on the sarcomere length $l=l_{0}\left(1+\gamma_{\mathrm{f}}\right)$, where $l_{0}$ is the average length in an unloaded resting state. Here $d_{0}=303.7033, d_{1}=-89.9638, d_{2}=-46.5338, d_{3}=-3.6730, e_{1}=$ $71.8140, e_{2}=-10.5776$ and $e_{3}=-7.6431$ are parameters of a truncated Fourier series fitted to match the experimental length-force relations reported in Strobeck \& Sonnenblick (1986). A plot of the active force is given in Fig. 4. 


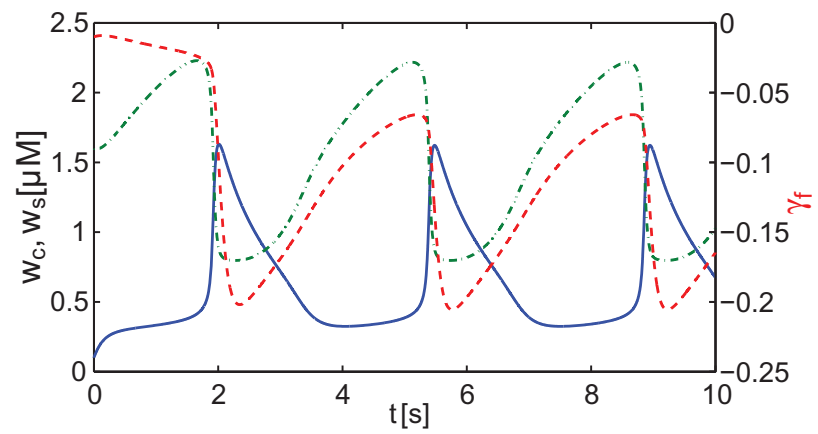

FIG. 5. Typical temporal progression of the proposed coupling of calcium concentrations $w_{\mathrm{c}}$ (solid), $w_{\mathrm{S}}$ (dashed-dotted) and mechanical activation $\gamma_{\mathrm{f}}$ (dashed) on a single material point, governed by (2.2) and (2.3).

The delay and amplitude of the calcium-activation interactions can be tuned with the specification (2.3). The described model corresponds to a phenomenological, only qualitative, representation of actin-myosin binding kinetics with typical timings and calcium function shapes (calcium sparks, calcium gradients and calcium waves leading to local excitations) in agreement with more accurate models coupling voltage and calcium kinetics. The reaction kinetics of calcium concentrations and active shortening of the sarcomeres regarded on a single material point behave as depicted in Fig. 5, where it is possible to see a delay of activation (red dashed line) with respect to the cytosolic calcium concentration which is qualitatively comparable with the results in Rice et al. (2008) (see also Iyer $e t$ al., 2004; Nobile et al. (2012)). On the other hand, the present model is not yet capable of correctly describing force-velocity relationships, as we neglect the microscopical information about sarcomere dynamics.

\subsection{Complete Lagrangian formulation of the model}

Summarizing, the following quasi-static system of coupled partial differential equations, written in a pure Lagrangian formulation, describes the interaction between displacements of the hyperelastic medium, mechanical activation and propagation of calcium concentrations:

$$
\begin{aligned}
-\operatorname{div}\left(J_{\mathrm{A}} \frac{\partial \mathcal{W}\left(\mathbf{F F}_{\mathrm{A}}^{-1}\right)}{\partial \mathbf{F}}-p \mathbf{F}^{-\mathrm{T}}\right) & =0, \\
J & =1, \\
\partial_{t} w_{\mathrm{c}}-\operatorname{div}\left(\mathbf{D}(\mathbf{F}) \nabla w_{\mathrm{c}}\right) & =K\left(w_{\mathrm{c}}, w_{\mathrm{s}}\right), \\
\partial_{t} w_{\mathrm{s}} & =L\left(w_{\mathrm{c}}, w_{\mathrm{s}}\right), \\
\partial_{t} \gamma_{\mathrm{f}} & =G\left(w_{\mathrm{c}}, \gamma_{\mathrm{f}}\right),
\end{aligned}
$$

in $\Omega \times(0, T)$. A sketch of the domain and boundaries is presented in Fig. 6 . The problem is equipped with suitable initial data for $w_{\mathrm{c}}, w_{\mathrm{s}}, \gamma_{\mathrm{f}}$ (see Table 3 ); the cytosolic calcium is subject to zero-flux boundary conditions,

$$
\mathbf{D}(\mathbf{F}) \nabla w_{\mathrm{c}} \cdot v=0 \quad \text { on } \Gamma,
$$




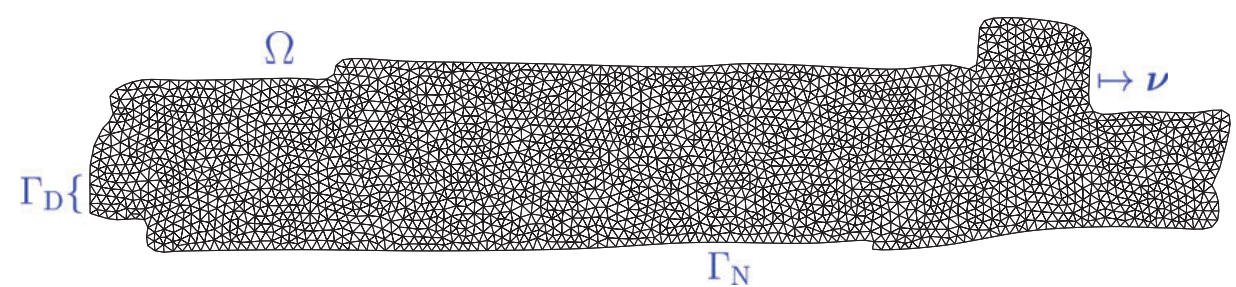

FIG. 6. Triangular mesh used in all numerical examples, consisting of 2781 nodes and 5560 elements. The cardiomyocyte dimensions are $\sim 109 \mu \mathrm{m} \times 26 \mu \mathrm{m}$. The 2D geometry has been reconstructed from confocal images of Srinivasan (2011). The sketch of the domain boundaries represents only the case when $\Gamma=\Gamma_{\mathrm{D}} \bigcup \Gamma_{\mathrm{N}}$.

whereas the boundary data for the mechanical problem are

$$
\begin{aligned}
\mathbf{u}=\mathbf{0} & \text { on } \Gamma_{\mathrm{D}}, \\
\mathbf{P} v=\mathbf{0} & \text { on } \Gamma_{\mathrm{N}}, \\
\mathbf{P} v+\alpha \mathbf{u}=\mathbf{0} & \text { on } \Gamma_{\mathrm{R}},
\end{aligned}
$$

where the boundary $\Gamma$ contains different regions for Dirichlet, Neumann and Robin conditions, respectively. In this way, we are able to reproduce different experimental conditions related to cell-cell and cell-matrix adhesions, in which (1) the displacement field is assumed to vanish on $\Gamma_{\mathrm{D}}$, meaning that the cell is fixed to a wall of the dish where the cell is located or it is in contact with a very rigid substrate; (2) stress-free conditions are assumed on $\Gamma_{\mathrm{N}}$ where $v$ is the normal vector to $\Gamma$, meaning that the cell has no contacts or it is located on a very compliant substrate; (3) Robin boundary conditions are imposed on $\Gamma_{\mathrm{R}}$, where $\alpha$ is a parameter representing the elastic response due to the presence of a surrounding tissue, i.e. contact with other myocytes, or fluid hindering and constraining the motion of the cell (see, e.g. Moireau et al., 2012). This last configuration is the most biophysically tunable even if more complex boundary conditions could be introduced in order to consider multiscale effects. (See Section 4.2 for a critical discussion.) In practice, for our numerical tests, whenever we apply Robin-type data, we do it on the whole boundary, i.e. $\Gamma_{\mathrm{R}}=\Gamma$.

The weak form of the equations (2.6) and (2.7) (needed to obtain approximate solutions using finite element methods) consists in finding at every $t>0,\left(\mathbf{u}, p, w_{\mathrm{c}}, w_{\mathrm{s}}, \gamma_{\mathrm{f}}\right) \in \mathbf{H}_{\Gamma_{\mathrm{D}}}^{1}(\Omega) \times L^{2}(\Omega) \times H^{1}(\Omega) \times$ $L^{2}(\Omega) \times L^{2}(\Omega)$ such that

$$
\begin{aligned}
& \int_{\Omega} \mathbf{P}\left(\mathbf{u}, p, \gamma_{\mathrm{f}}\right): \nabla \mathbf{v}=0, \quad \int_{\Omega} q(J(\mathbf{u})-1)=0, \\
& \frac{\partial}{\partial t} \int_{\Omega} w_{\mathrm{c}} \phi_{c}+\int_{\Omega} \mathbf{D}(\mathbf{u}) \nabla w_{\mathrm{c}} \cdot \nabla \phi_{c}-\int_{\Omega} K\left(w_{\mathrm{c}}, w_{\mathrm{s}}\right) \phi_{c}=0, \\
& \frac{\partial}{\partial t} \int_{\Omega} w_{\mathrm{s}} \phi_{s}-\int_{\Omega} L\left(w_{\mathrm{c}}, w_{\mathrm{s}}\right) \phi_{s}=0, \\
& \frac{\partial}{\partial t} \int_{\Omega} \gamma_{\mathrm{f}} \phi_{f}-\int_{\Omega} G\left(w_{\mathrm{c}}, \gamma_{\mathrm{f}}\right) \phi_{f}=0
\end{aligned}
$$

for all test functions $\left(\mathbf{v}, q, \phi_{c}, \phi_{s}, \phi_{f}\right) \in \mathbf{H}_{\Gamma_{\mathrm{D}}}^{1}(\Omega) \times L^{2}(\Omega) \times H^{1}(\Omega) \times L^{2}(\Omega) \times L^{2}(\Omega)$, where $\mathbf{H}_{\Gamma_{\mathrm{D}}}^{1}(\Omega):=\left\{\mathbf{v} \in \mathbf{H}^{1}(\Omega):\left.\mathbf{v}\right|_{\Gamma_{\mathrm{D}}}=\mathbf{0}\right\}$. 
To approximate this problem, we use a Galerkin finite element discretization, which results in a system of nonlinear, coupled differential equations. In view of the discrete inf-sup (or LBB) condition for mixed problems (Quarteroni \& Valli, 1997) such as the linearized elasticity equations coming from (2.6), we employ elements of the Taylor-Hood type, i.e. piecewise quadratic finite elements for the spatial approximation of displacements, whereas the discretization of all other fields is accomplished using continuous piecewise linear elements. A Newton method is used for the set of nonlinear equations (see Appendix), and the resulting linear systems are solved using the GMRES method with a Cahouet-Chabard preconditioner (see, e.g. Quarteroni \& Valli, 1997). A Picard (fixed-point) algorithm is employed for the coupling, i.e. we first solve the nonlinear mechanics for fixed calcium concentrations and activation and the second step consists in solving the reaction-diffusion system for $w_{\mathrm{c}}, w_{\mathrm{s}}, \gamma_{\mathrm{f}}$ for a fixed displacement. If needed, this process is repeated until a given tolerance is reached. Such a strategy has been chosen basically to preserve modularity at the implementation level. Certainly, a full monolithic approach (see Wong et al., 2013, and the references therein) is also feasible. The time interval $(0, T)$ is discretized into subintervals $\left[t^{n}, t^{n+1}\right], n=0,1, \ldots$, with $t^{n}=n \Delta t$ where $\Delta t=0.01 \mathrm{~ms}$ is a fixed timestep. The time integration is performed using an implicit Euler method.

\section{Numerical results}

The spatial convergence of the finite element method for the coupled system is first studied by considering the following steady version of problem (2.6):

$$
\begin{aligned}
-\operatorname{div}\left(a\left(1+\gamma_{\mathrm{f}}\right) \mathbf{F}-a g\left(\gamma_{\mathrm{f}}\right) \mathbf{F} \mathbf{f}_{0} \otimes \mathbf{F} \mathbf{f}_{0}-p \mathbf{F}^{-\mathrm{T}}\right) & =\mathbf{0}, \\
J & =1, \\
-\operatorname{div}\left(\mathbf{D}(\mathbf{F}) \nabla \gamma_{\mathrm{f}}\right)+v_{4} \gamma_{\mathrm{f}} & =j\left(w_{\mathrm{s}}\right),
\end{aligned}
$$

imposing in (2.1) the parameters $b=0$ and $a_{f}=0$ on the dimensional domain $\Omega=(0,1)^{2}$ and where $j\left(w_{\mathrm{s}}\right)=\left(d_{1} v_{5} / d_{2}\right) w_{\mathrm{s}}-\left(d_{1} v_{1} / d_{2}\right)$. We compare the numerical results with the following exact solution of (3.1):

$$
\mathbf{u}=\left(-\frac{\lambda}{\lambda+1} x, \lambda y\right)^{\mathrm{T}}, \quad p=a \frac{\left(1+\gamma_{\mathrm{f}}\right)^{2}}{(1+\lambda)^{2}}, \quad \gamma_{\mathrm{f}}=c \sin x
$$

where we set $\lambda=0.1, c=0.1$ and impose boundary conditions as follows:

$$
\begin{gathered}
\mathbf{u} \cdot v=0 \quad \text { on }[0,1] \times\{0\} \cup\{0\} \times[0,1], \\
\mathbf{P} v= \begin{cases}a\left(\frac{\left[(1+\lambda)^{4}-\left(1+\gamma_{\mathrm{f}}\right)^{4}\right]}{(1+\lambda)^{3}\left(1+\gamma_{\mathrm{f}}\right)^{2}}, 0\right) & \text { on }[0,1] \times\{1\}, \\
\mathbf{0} & \text { on }\{1\} \times[0,1],\end{cases} \\
\mathbf{D}(\mathbf{F}) \nabla \gamma_{\mathrm{f}} \cdot v= \begin{cases}0 & \text { on }[0,1] \times\{0,1\}, \\
(1+\lambda)^{2} D_{11} \cos x v_{x} & \text { on }\{0,1\} \times[0,1] .\end{cases}
\end{gathered}
$$

This preliminary system is solved monolithically with a quasi-Newton method, linearizing with respect to displacements $\mathbf{u}$ and pressure $p$ only. This quasi-Newton scheme features linear convergence and the total number $n_{q N}$ of iterations depends on the initial guess. We have employed $\left(\mathbf{u}, p, \gamma_{\mathrm{f}}\right)=(\mathbf{0}, 0,0)$, 
TABLE 2 Mesh-size, computed errors in $H^{1}$ - and $L^{2}$-norms and observed convergence rates for the numerical approximation of the steady-state problem (3.1) with $\mathbb{P}_{2}-\mathbb{P}_{1}-\mathbb{P}_{1}$ finite elements

\begin{tabular}{lcccccccc}
\hline$h$ & $e_{1}(\mathbf{u})$ & $r_{1}(\mathbf{u})$ & $e_{0}(p)$ & $r_{0}(p)$ & $e_{1}\left(\gamma_{\mathrm{f}}\right)$ & $r_{1}\left(\gamma_{\mathrm{f}}\right)$ & $e_{T}$ & $r_{T}$ \\
\hline 0.1 & $2.298107 \mathrm{e}-4$ & - & $2.723865 \mathrm{e}-1$ & - & 1.54062163 & - & 1.81323797 & - \\
0.05 & $5.104010 \mathrm{e}-5$ & 2.170743 & $1.410617 \mathrm{e}-1$ & 0.949328 & $7.671277 \mathrm{e}-1$ & 1.005973 & $9.082405 \mathrm{e}-1$ & 0.997422 \\
0.025 & $1.211586 \mathrm{e}-5$ & 2.074733 & $7.172121 \mathrm{e}-2$ & 0.975855 & $3.827318 \mathrm{e}-1$ & 1.003133 & $4.544651 \mathrm{e}-1$ & 0.998905 \\
0.0125 & $2.956262 \mathrm{e}-6$ & 2.035051 & $3.615468 \mathrm{e}-2$ & 0.988217 & $1.911534 \mathrm{e}-1$ & 1.001603 & $2.273110 \mathrm{e}-1$ & 0.999502 \\
0.00625 & $7.304491 \mathrm{e}-7$ & 2.016918 & $1.815042 \mathrm{e}-2$ & 0.994180 & $9.552305 \mathrm{e}-2$ & 1.000810 & $1.136742 \mathrm{e}-1$ & 0.999763 \\
\hline
\end{tabular}

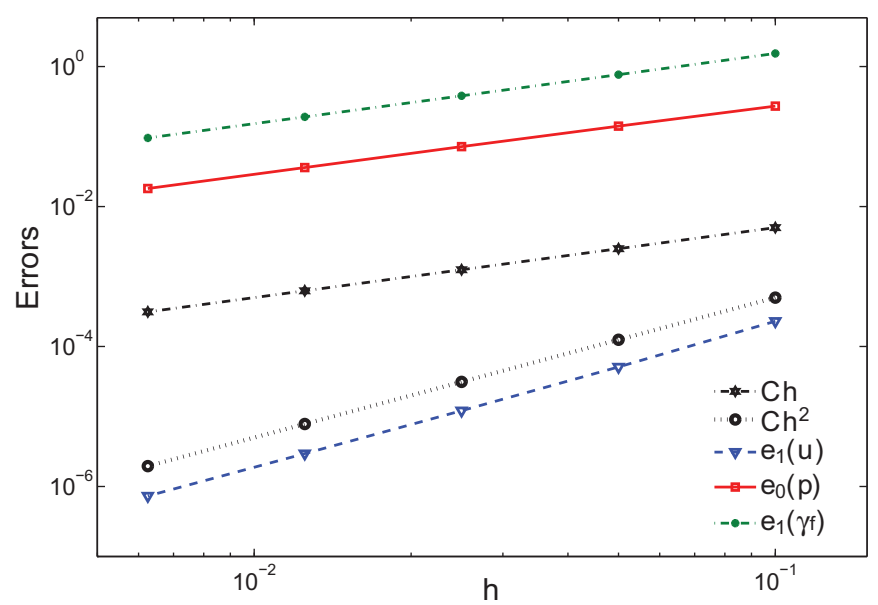

FIG. 7. Mesh-size and computed errors in $H^{1}$ - and $L^{2}$-norms for the numerical approximation of the steady-state problem (3.1) with $\mathbb{P}_{2}-\mathbb{P}_{1}-\mathbb{P}_{1}$ finite elements.

leading to $n_{q N}=16$. The unknowns $\left(\mathbf{u}, p, \gamma_{\mathrm{f}}\right)$ are discretized using $\mathbb{P}_{2}-\mathbb{P}_{1}-\mathbb{P}_{1}$ elements. By

$$
e_{m}(\mathbf{u}):=\left|\mathbf{u}-\mathbf{u}_{h}\right|_{\boldsymbol{H}^{m}(\Omega)} /|\mathbf{u}|_{\boldsymbol{H}^{m}(\Omega)},
$$

we denote relative errors in the $H^{m}$-semi-norm and by $r_{m}(\mathbf{u})$ we denote the experimental rate of convergence between two consecutive meshes, one with half meshsize $h$. Table 2 and Fig. 7 report the convergence history for the coupled problem, where convergence rates of $\mathcal{O}(h)$ are observed for $\gamma_{\mathrm{f}}, w_{\mathrm{s}}$ in $H^{1}$ and for $p$ in $L^{2}$, while for $\mathbf{u}$ the convergence rate in the $H^{1}$-norms is of order $\mathcal{O}\left(h^{2}\right)$. In the energy norm the total relative error $e_{T}$ is defined as the sum of the relative errors and the total order of convergence $r_{T}$ is the minimum order among $r_{m}(\mathbf{u}), r_{m}(p)$ and $r_{m}\left(\gamma_{\mathrm{f}}\right)$.

We now provide a set of numerical experiments demonstrating some desirable features of the mathematical model of transient mechano-chemical interactions. Confocal images of a single adult rat cardiomyocyte in a stress-free/strain-free configuration (Srinivasan, 2011) are employed to create a computational domain from which a triangular mesh $\mathcal{T}_{h}$ of 2781 nodes and 5560 linear elements (see Fig. 6) was carefully generated using the open source mesh manipulator Gmsh (Geuzaine \& Remacle, 2009). The main axis of the cell has an approximate length of $109 \mu \mathrm{m}$, while its diameter is about $26 \mu \mathrm{m}$. The (passive) hyperelastic behaviour of the cell will be represented by setting in (2.1) the parameters $b=0$ and $a_{f}=0$ for the examples based on the confocal microscopy of the isolated cardiomyocyte and a 
transversely isotropic material law will be used in section 3.4. The semi-discrete problem will be solved using the procedure illustrated in Algorithm 1.

A preferred direction of contraction for the $2 \mathrm{D}$ cases has been initially set to $\mathbf{f}_{0}=(1,0)^{\mathrm{T}}$. Such an assumption is inspired by the fact that the activation is generated along the direction given by the sarcomeres' orientation (Tracqui et al., 2008). If we regard a compound of cells instead, we may assume that the myocytes are aligned along the direction of the collagen fibres (Stålhand et al., 2011). We specify

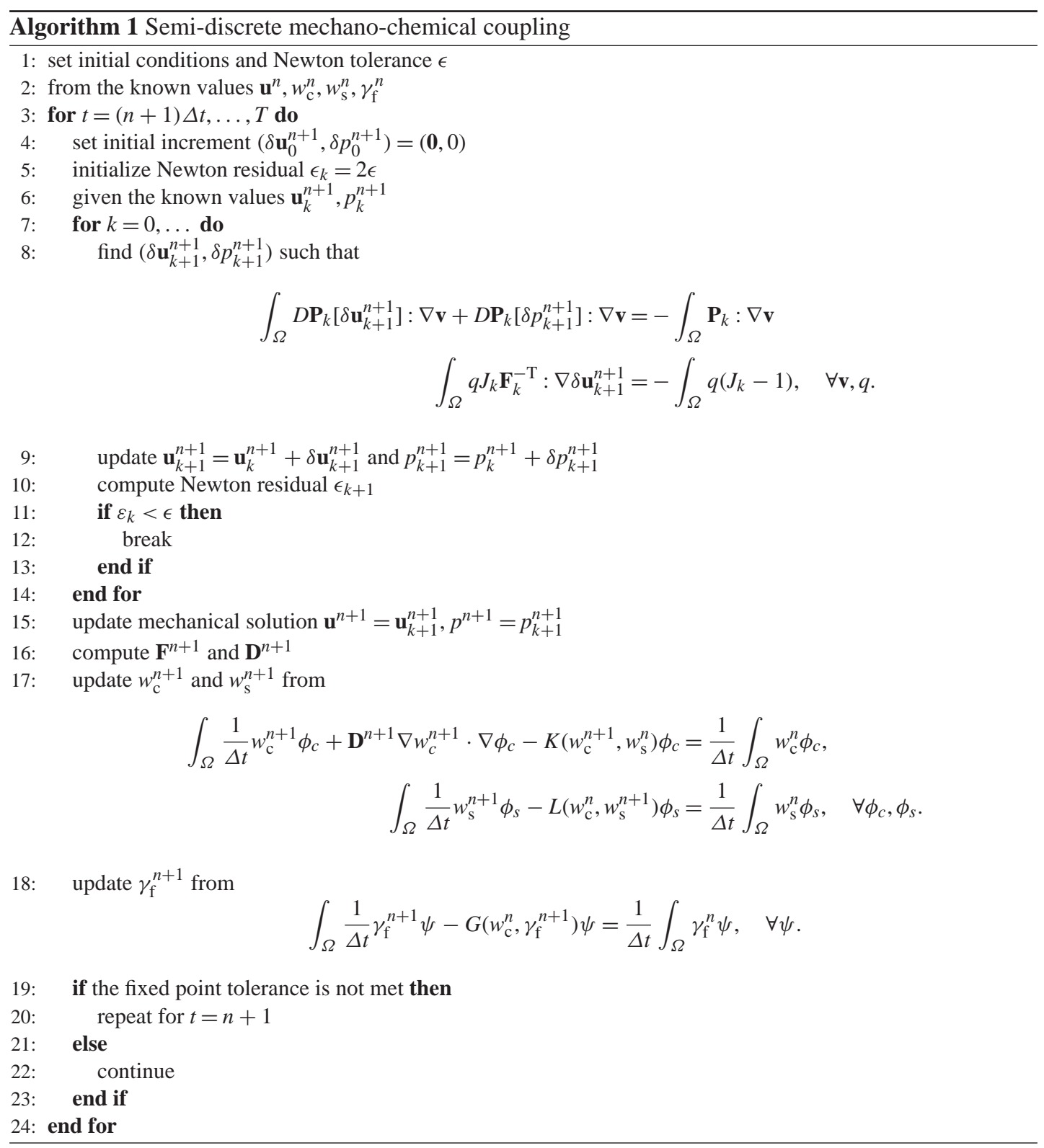


TABLE 3 Initial conditions for Examples 1-4

\begin{tabular}{lcc}
\hline Variable & Value & Unit \\
\hline $\mathbf{u}$ & 0 & $\mu \mathrm{m}$ \\
$\gamma_{f}$ & -0.001 & - \\
$w_{\mathrm{c}}$ & 0.1 & $\mu \mathrm{M}$ \\
$w_{\mathrm{s}}$ & 1.6 & $\mu \mathrm{M}$ \\
\hline
\end{tabular}

initial data for displacements, calcium concentrations and activation variables as in Table 3 that agree with the tests in Tracqui \& Ohayon (2009).

\subsection{Boundary conditions effects}

The simulations of self-sustained chemical and mechanical interactions are initiated with a protocol of spark of cytosolic calcium near the nucleus of the cell (Iribe et al., 2009). As in Tracqui et al. (2008) and Tracqui \& Ohayon (2009), these are incorporated through an instantaneous increase in the parameter $v_{1}$ (we use $v_{1,0}=2.45 v_{1}$ ) on the desired location. These correspond to the smallest values that are able to onset the calcium wave propagation.

In this first example, we discuss the importance of boundary conditions on the overall dynamics emerging from the active-strain coupling, thus mimicking different physiological and/or experimental scenarios under which the single myocyte could be studied. We present two simulations where the displacements $\mathbf{u}$ are (1) of Robin type (left panels) on $\Gamma$ with Robin coefficient $\alpha=1 \times 10^{-2}$; (2) of Dirichlet type (right panels), setting zero displacements on the left part of the boundary $\Gamma_{\mathrm{D}}$ (see Fig. 6) and letting $\Gamma_{\mathrm{N}}$ with stress-free boundary conditions (spring boundary data).

In Fig. 8, we observe the propagation of $w_{\mathrm{c}}$ towards the extremities of the cell comparing the two sets of boundary conditions. In accordance with Fig. 5, we also see that the activation causes a contraction of the cardiomyocyte. Already from the second pair of snapshots, the calcium propagation is qualitatively different in both cases and the second final activation at later times confirms such a difference. The propagating wave presents asymmetric maxima, and for case 2 in particular, a higher calcium wave moves towards the fixed boundary. In general, however, a calcium accumulation is obtained at the corners of the geometry.

The mechanical response presents differences in the shape of the deformations (see Fig. 9 as well). Fixing the left part of the cell, one obtains an eventual bending of the myocyte (reached at the maximum contracted state) that cannot be appreciated in the case of the spring boundary data. This numerical example also points to the difficulty of predicting the exact motion of a single cardiomyocyte, since in experimental settings it is observed to take place in 'unexpected' directions due to the irregularity of gap junctions, or location and size of adhesion regions. However, some characteristic motions can be recovered in our case, such as movement with respect to the principal direction and bending (Delbridge \& Roos, 1997).

A careful inspection of the velocity field in Fig. 8 and of the boundary motion in Fig. 9 highlights the characters of the movement, with an almost perfect symmetry for the spring boundary case. In addition, the model is able to reproduce the experimental values of the calcium conduction velocity as shown in Fig. 10 . 

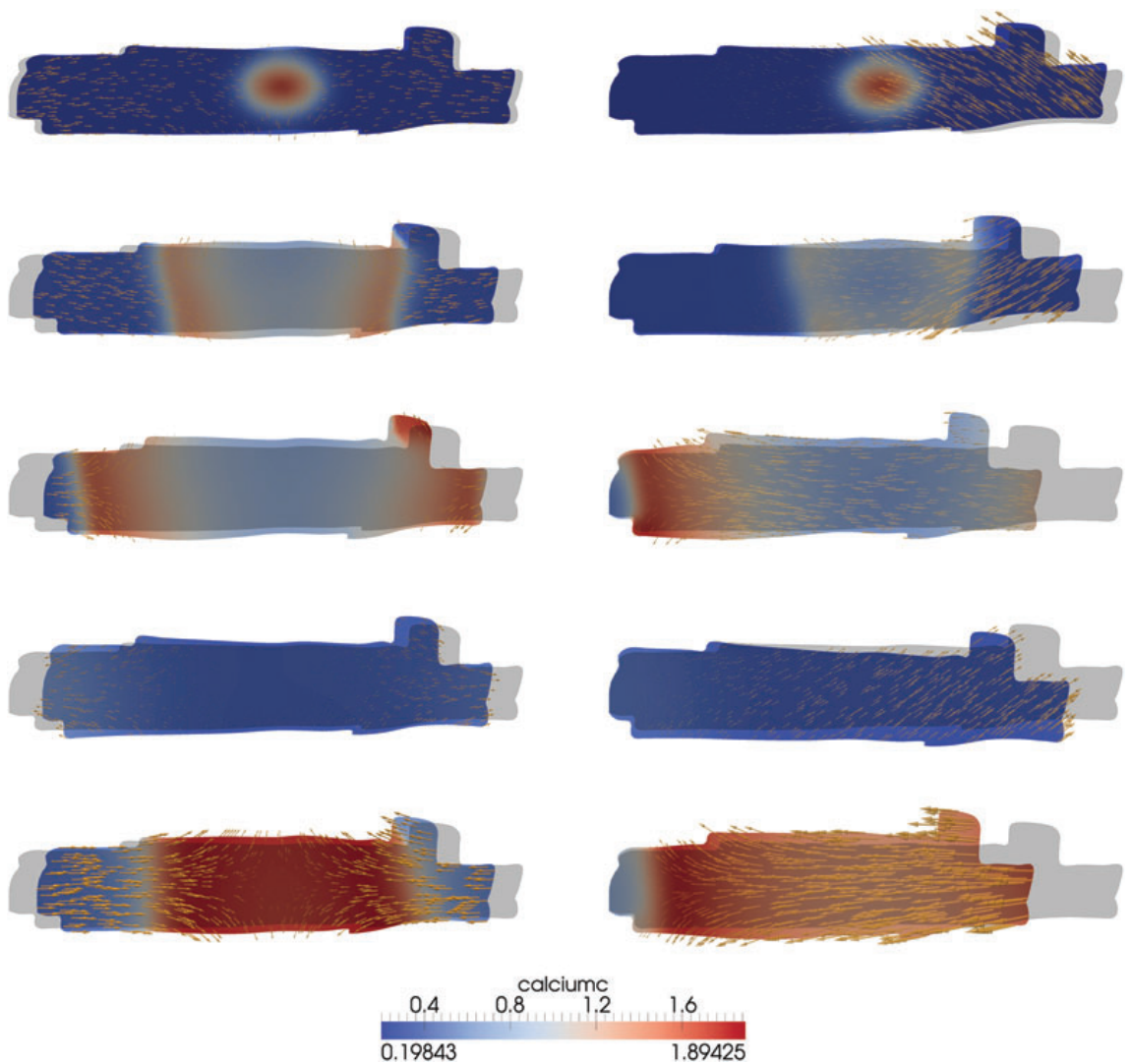

FIG. 8. Example 1: Snapshots of the propagation of cytosolic calcium on the deformed configuration for times $t=$ $0.2,1.0,2.1,3.0,4.0 \mathrm{~ms}$ (from top to bottom), for Robin boundary conditions (left panels) and Dirichlet data (right panels). The grey surface in each plot depicts the cell domain in the reference configuration. The colour code refers to the cytosolic calcium concentration and the arrows represent the velocity field.

\subsection{Calcium sparks initiated at different regions}

Now we turn to the study of different stimulation regions to assess the importance of spatial localization of $\mathrm{Ca}^{2+}$ release in determining the overall physiological output of a myocyte. We will stick to the case of Robin boundary data as defined in the previous section. In the first test, we apply a calcium spark originated from the left part of the cell and we observe a calcium wave propagating from left to right (see Fig. 11). In all cases, we found a mean contraction amplitude of about $11 \mu \mathrm{m}$ and a mean contraction duration of $1.2 \mathrm{~s}$.

The second test case we perform consists in initiating two calcium waves from the opposite sides of the cell. The observed behaviour corresponds to the collision (annihilation) of the propagating waves at the cell centre (see Fig. 12), as reported in the experiments by Ishide et al. (1990) and Zimmermann \& Walz (1997). We also see that the pressure remains controlled in both cases, but, as expected, it accumulates near the zones of irregular boundaries.

In our third test case, we perform a double stimulation by calcium sparks from a region near the centre (with an initial increase $v_{1,0}=2.45 v_{1}$ ) and one side of the cell (with an initial increase $\tilde{v}_{1,0}=1.3 v_{1}$ ). 

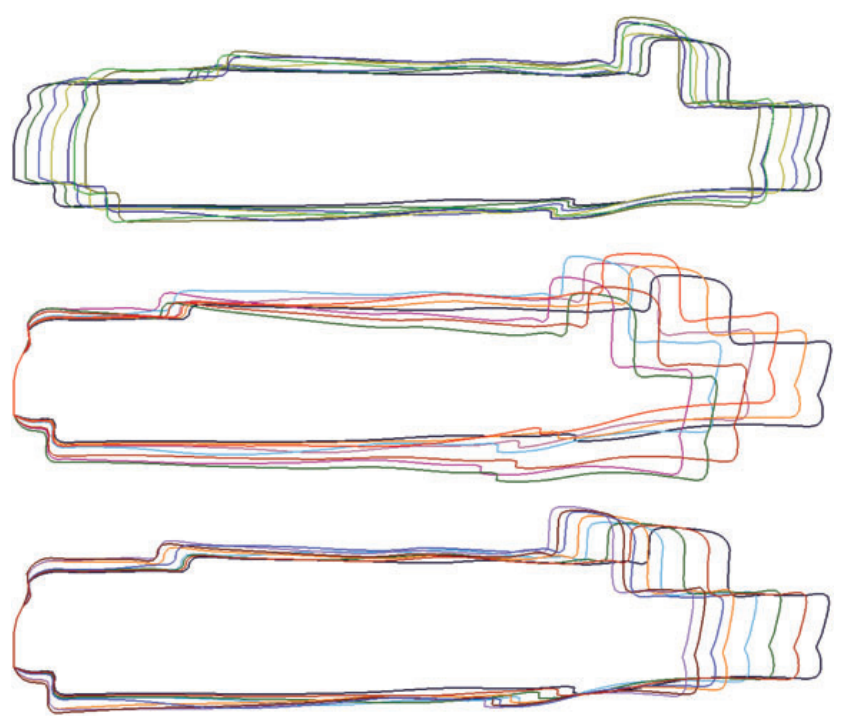

FIG. 9. Example 1: Cell motion for pure Robin (top), Dirichlet-Neumann (middle) and Dirichlet-Robin (bottom) boundary conditions. The snapshots are taken at times $t=0.2,0.6,1.0, \ldots, 3.0 \mathrm{~ms}$.
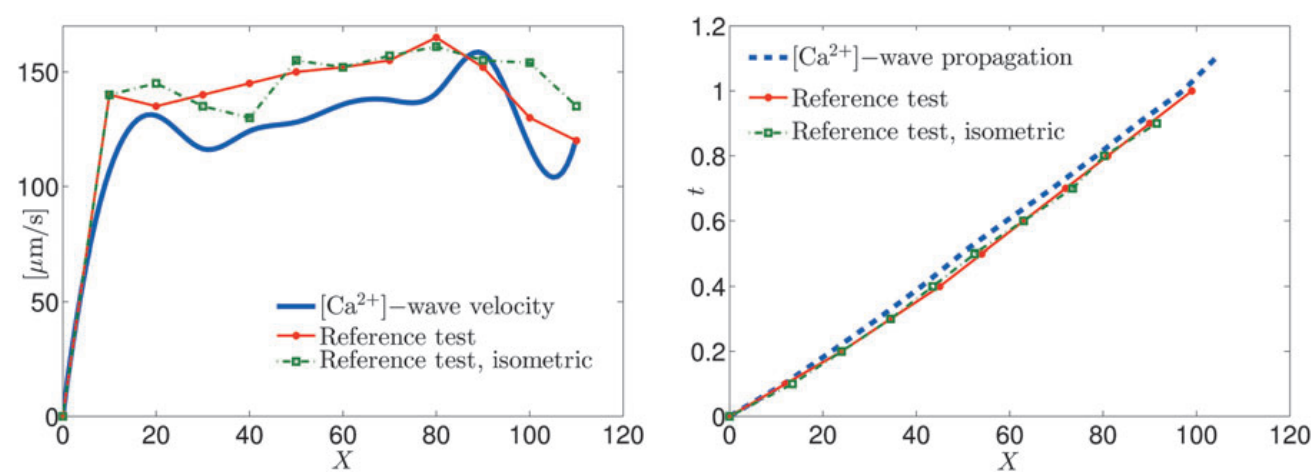

FIG. 10. Example 3: Local variation in calcium wave propagation velocity (thick line) and comparison with results from Okada et al. (2005) for unloaded (thin line) and isometric (thin line) tests (left). Propagation of the $\left[\mathrm{Ca}^{2+}\right]$ waves (thick dashed line) and comparison with unloaded (thin line) and isometric (thin line) tests from Okada et al. (2005) (right).

Such a setting simulates the calcium release from the nucleus and its entering from outside in the case of coupling with a neighbouring cell. In Fig. 13, we see that the stronger wave coming from the nucleus completely overtakes the wave starting from the left side, the latter being hardly noticeable already at early times.

\subsection{Analysis of stresses and calcium velocities}

In Fig. 14, we present a set of snapshots of the trace of the Cauchy stress tensor and the principal stretch. Robin boundary conditions are employed for the first set of plots, where we observe complex stress 

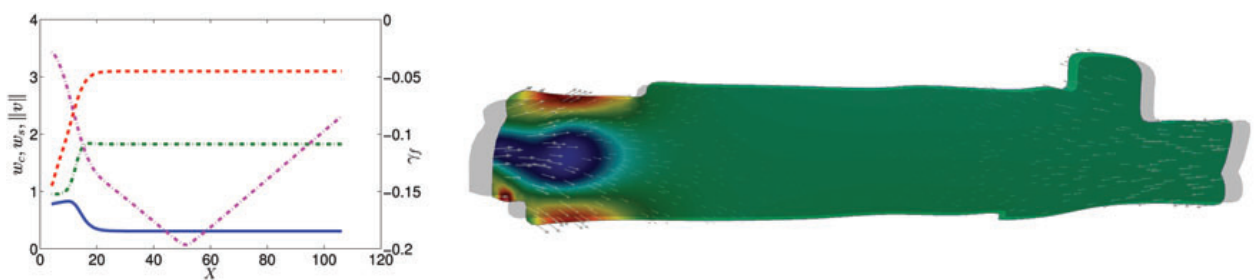

(a)
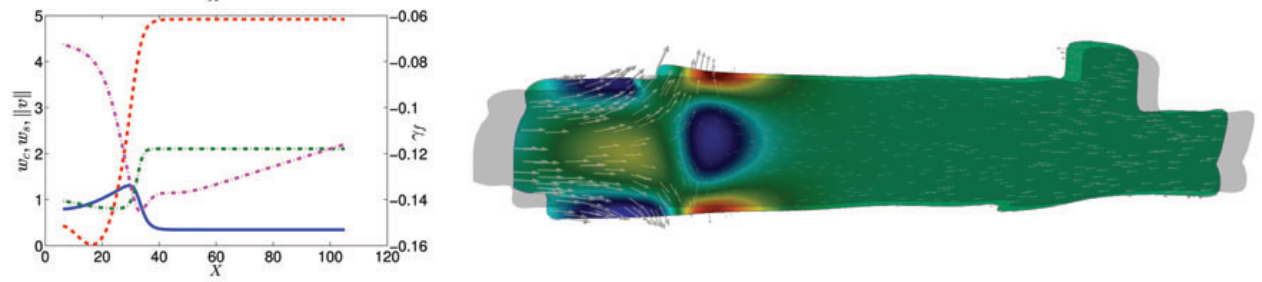

(b)
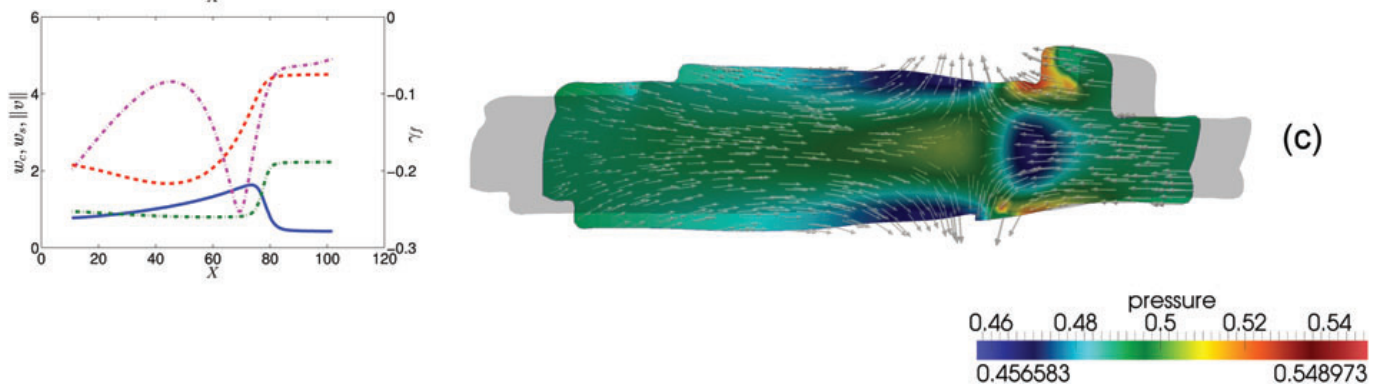

FIG. 11. Example 2a: Single calcium spark initiated on the left part of the cell. Snapshots of calcium concentrations $w_{\mathrm{c}}$ (solid), $w_{\mathrm{S}}$ (dashed-dotted), rescaled mechanical activation $\gamma_{\mathrm{f}}$ (dashed) and velocity norm (dashed-dotted) computed along the main axis of the cell (left panels) and pressure and velocity fields (right panels), at times $t=1.0,3.0,4.0 \mathrm{~ms}(\mathrm{a}, \mathrm{b}$ and c).

patterns only in the proximity of the stimulation region. On the other hand, when applying boundary data of Dirichlet type, we can readily see regions of stress and stretch accumulation near the fixed boundary and near the boundary irregularities. We obtained an average propagation velocity of $123 \mu \mathrm{m} / \mathrm{s}$ for the calcium wave. A comparison with results from Okada et al. (2005) in terms of local variation in the calcium wave propagation velocity (computed as the ratio between the local maximal calcium gradient and the time needed to reach the corresponding local maximal distance) and propagation of calcium is presented in Fig. 10. We see that the velocity of propagation of the wavefront increases along the cell length.

\subsection{Active contraction on a cylinder}

Finally, we test our model in its full complexity and we close with a series of computations performed on an idealized myocyte represented by a cylinder of size $24 \times 24 \times 120 \mu \mathrm{m}$. Here, the hyperelastic behaviour of the cell is modelled by the anisotropic law (2.1) where the parameters are chosen as in Holzapfel \& Ogden (2009): $a_{f}=15.193 \mathrm{KPa}, b=20.417, b_{f}=11.176$. The computational mesh consists of 9486 vertices, 4660 triangles (lying on the boundaries) and 49500 tetrahedral elements. We simulate the propagation of a calcium wave and its interaction with mechanical response using the same parameters as in the previous examples. The sarcomere directions are initially chosen as $\mathbf{f}_{0}=(0,0,1)^{\mathrm{T}}$. The elastic body is fixed on the boundary $z=0$ and stress-free conditions are imposed 
(a)
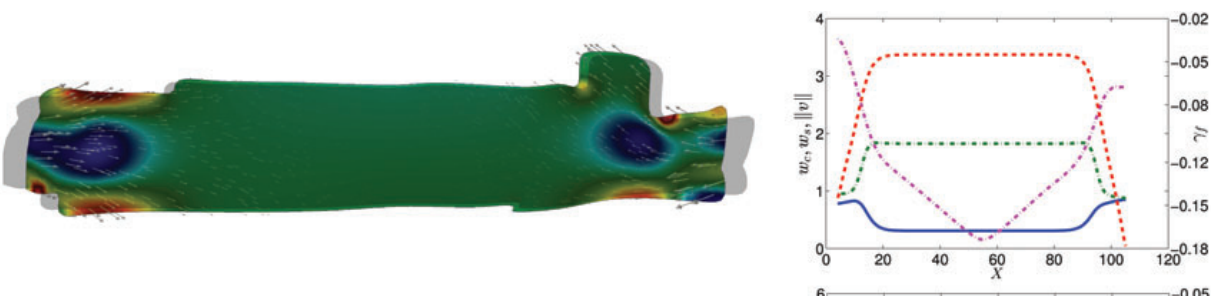

(b)
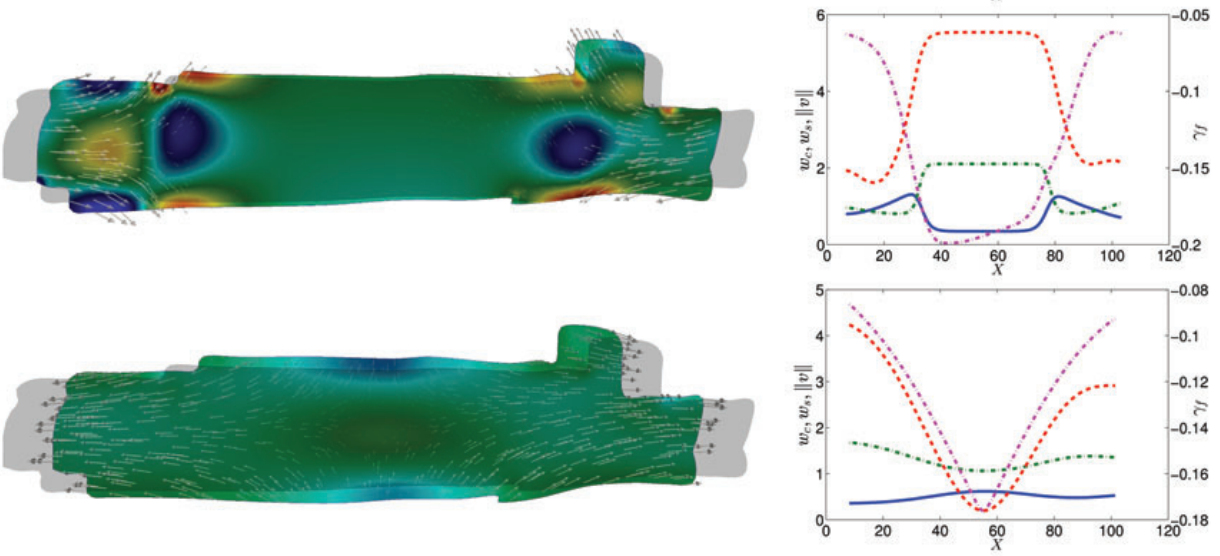

(c)
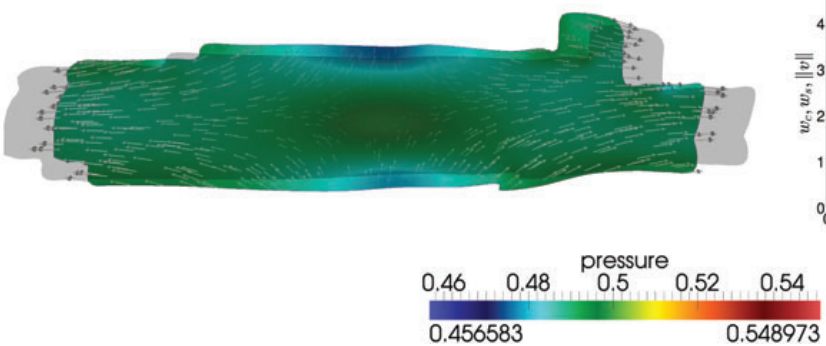

FIG. 12. Example 2b: Two calcium sparks initiated at both ends of the cell. Snapshots of pressures and velocities (left panels), and calcium concentrations $w_{\mathrm{c}}$ (solid), $w_{\mathrm{s}}$ (dashed-dotted), rescaled mechanical activation $\gamma_{\mathrm{f}}$ (dashed) and velocity norm (dasheddotted) computed along the main axis of the cell (right panels), at times $t=1.0,3.0,4.0 \mathrm{~ms}$ (a, b and c).
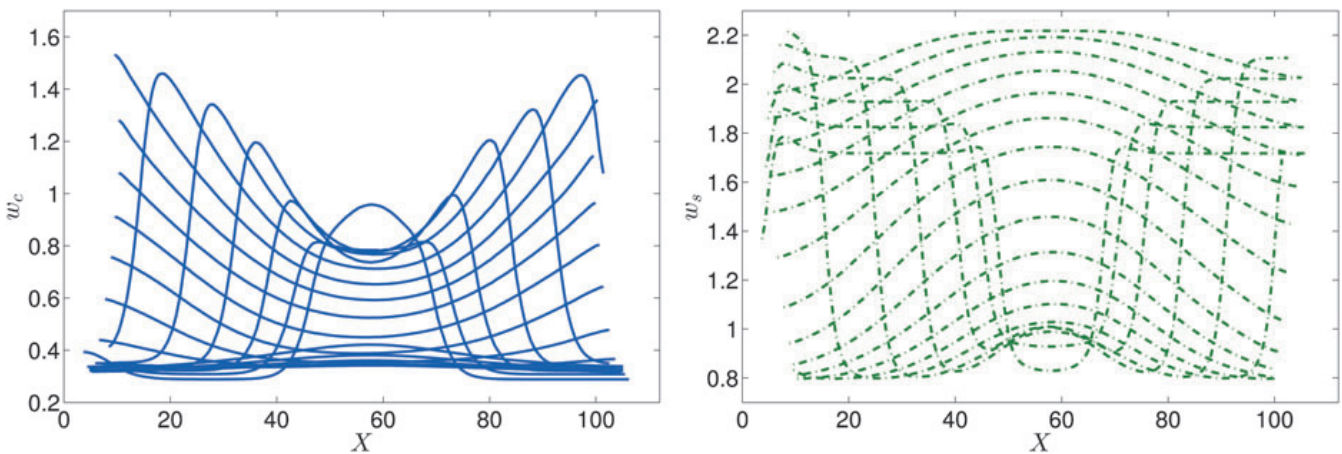

FIG. 13. Example 2c: Two calcium sparks initiated at the nucleus and left end of the cell. Snapshots of the propagation of calcium concentrations $w_{\mathrm{c}}$ (left) and $w_{\mathrm{s}}$ (right) along the main axis of the cell at times $t=0.2,0.4, \ldots, 3.4,3.6 \mathrm{~ms}$.

elsewhere (see Fig. 15). As in Example 1, we observe a bending behaviour, also in accordance with simulations in Tracqui \& Ohayon (2009), and in general expected for an fibre-dependent activation (see Rossi et al., 2012). 
(a)
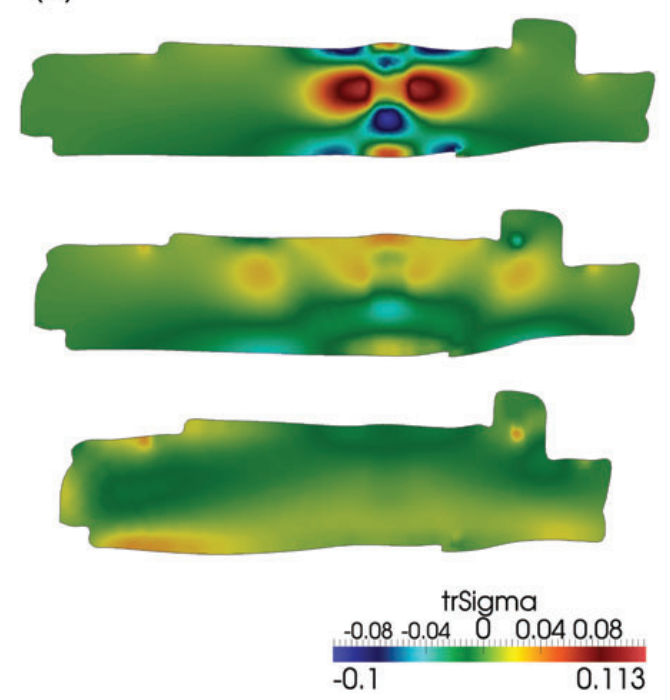

(b)
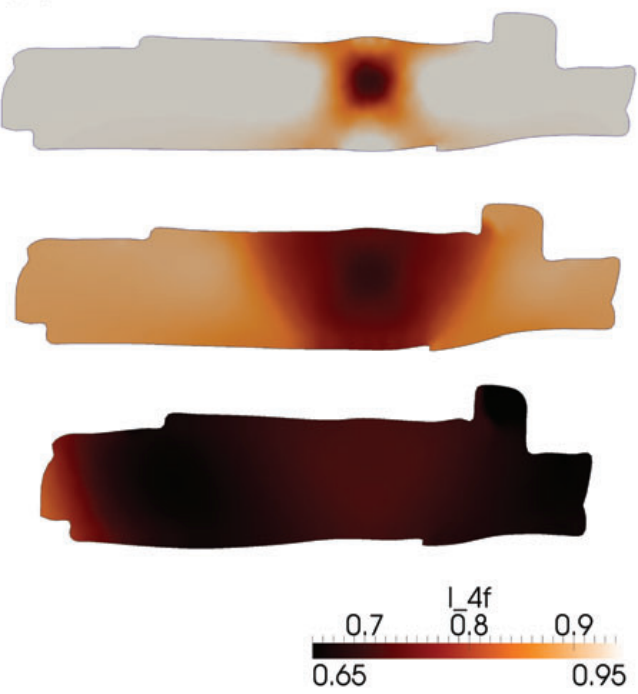

FIG. 14. Example 3: Snapshots of the trace of the Cauchy tensor $\operatorname{tr}\left(\mathbf{P F}^{\mathrm{T}}\right)$ (a), and principal stretches $I_{4, f}(\mathrm{~b})$ plotted on the deformed domains at times $t=0.1,0.4,0.8 \mathrm{~ms}$ (from top to bottom).
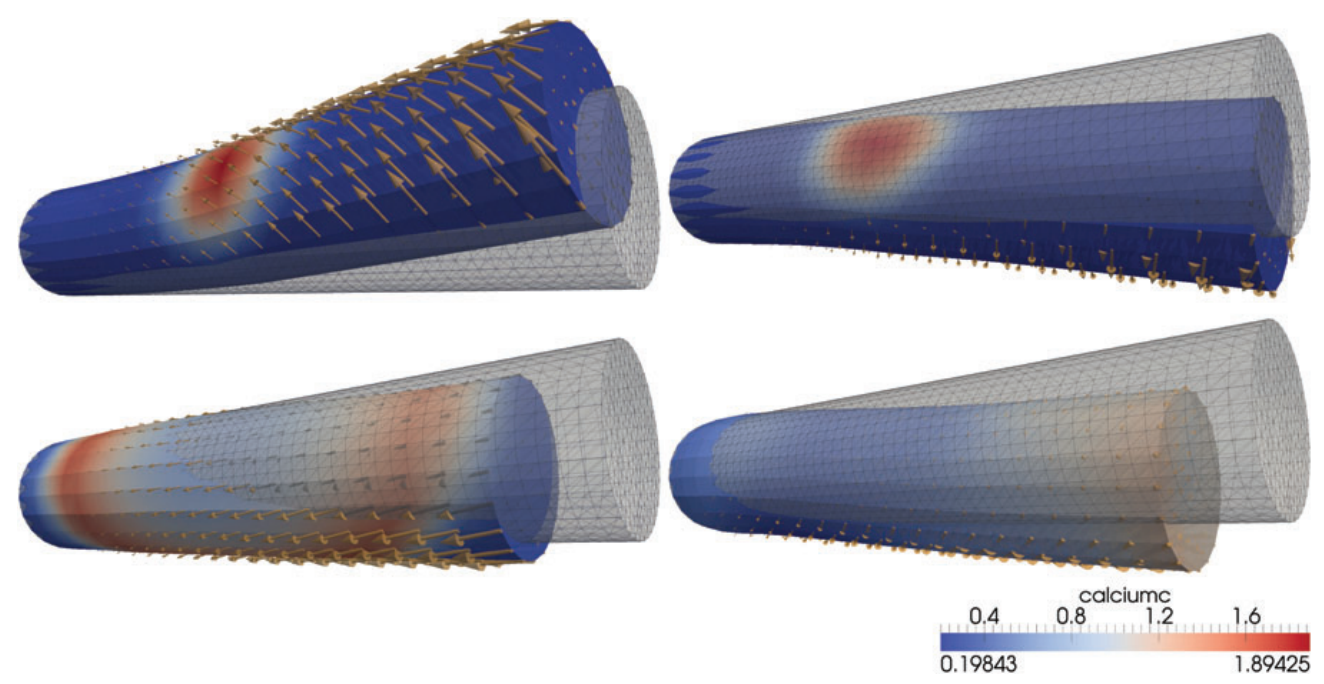

FIG. 15. Example 4: From top left to bottom right, snapshots of a deformable cylinder and calcium concentration $w_{\mathrm{c}}$ at time $t=0.17,0.25,1.23,2.68 \mathrm{~ms}$.

\section{Discussion \& concluding remarks}

We have presented a mathematical model of the mechano-chemical coupling in individual cardiomyocytes based on an active-strain approach (Ruiz-Baier et al., 2013). The proposed activation mechanism 
is consistent with a thermodynamic framework, as derived in Stålhand et al. (2011), and it entails a nonlinear interaction among calcium dynamics and local stretches. Our continuum approach is on the line of recent bio-chemo-mechanical models of single cells (Deshpande et al., 2006, 2008; Ronan et al., 2012) even if active-strain hyperelasticity has been adopted in this work. Our model reproduces the propagation of calcium waves and the corresponding spontaneous contraction interacting within the cell (Takamatsu et al., 1990). A finite element method is used to discretize the model equations; a set of numerical experiments give evidence of the main features of the model and its ability in predicting calcium propagation patterns and contractility in good agreement with experimental observations.

\subsection{Experimental vs. modelled boundary conditions}

Experimental evidences in adult rat myocyte demonstrated how the spatial segregation of cell-matrix and cell-cell adhesions to myocyte borders have fundamental effects for effective electromechanical coupling and arrhythmogenesis (Li et al., 2006). From the biomechanical point of view, such adhesions play the role of specialized and localized boundary conditions, anchoring cells and tissues to the extracellular matrix. However, being mechanosensitive in the cardiomyocytes, they act as tunable constraints modifying the stress concentration according to the cell function and modulating tissue organization. The effects of boundary conditions on cardiomyopathies have been experimentally well recognized by increased fibrosis and tissue stiffening (Berry et al., 2006; Goktepe et al., 2010). In fact diseased myocardium is usually studied throughout interaction between cells and stiff substrates, analysing the increase in force generation, remodelling and adhesion. Extracellular space conditions have been further hypothesized to potentiate the organization of cytoskeletal scaffolds and the contractile response as well (Grosberg et al., 2011). Moreover, focal adhesions have been recently proposed to mechanically stabilize myocyte form and functions (McCain et al., 2012) by analysing stiff micro-environment effects on the intrinsic myocyte loading.

In this work, we studied the boundary conditions effects on the overall myocyte dynamics. In particular, we simulated self-sustained chemical and mechanical interactions mimicking different physiological and/or experimental scenarios via Robin and Dirichlet boundary conditions: i.e. fixed regions of the geometrical boundary, representing adhesion regions (Delbridge \& Roos, 1997) or spring-like boundaries, reproducing the presence of contact myocytes.

A careful inspection of calcium wave propagation and stress analysis confirmed several experimental evidences: (i) calcium activation timing and conduction velocities; (ii) asymmetric calcium maxima and stress concentrations; (iii) myocyte bending and driving contraction due to internal calcium sparks.

\subsection{Limitations and perspectives}

Several limitations of this study should be mentioned. First, we point out that a critical aspect to obtain physiological displacements of the cell is the correct treatment of boundary conditions other than a careful representation of its anisotropic internal structure, its external geometry and its nonlinear chemical dynamics. In the novel chemo-mechanic approach presented here, we have shown that Robin boundary conditions can reproduce better the experimental observations, but there is still the issue of tuning the Robin coefficient $\alpha$ in (2.7).

From the mechanical point of view an effective alternative (Laadhari et al., 2012) is to consider a Eulerian description of a fluid-structure interaction problem where actual fluid is modelled outside the elastic cell, and capturing the interface using a level-set approach. 
Other intracellular microstructures, i.e. intercalated discs, should be taken into account for a more accurate geometric model. However, the complex multiscale phenomena involved and the lack of knowledge of the specific mechanical properties of such structures require the fine tuning of simplified cellular models. In this perspective, the model proposed here represents an alternative approach in terms of continuum mechanics theory.

From a chemical viewpoint, however, more complex boundary conditions should be introduced in order to represent complete membrane ionic exchanges other than calcium, i.e. $\mathrm{Na}$ and $\mathrm{K}$. The role of gap junctions has been discarded in the present study. However, it would be crucial to capture a correct propagation of electrical activation in non-isolated cardiac myocytes and the whole cardiac tissue, since it provides a physiological description of the anisotropic electrical coupling. In addition, the contractile dynamics of cardiomyocytes depends strongly on their spatial localization (ventricular, atrial, epi, endo) and on the end terminal effects (McCain et al., 2012), which suggests that time delays of excitation should be also considered.

A more accurate modelling of the calcium dynamics itself (here limited to two calcium concentrations only) by introducing the Sodium-Calcium Exchanger and the NCX-NKA system, e.g. would help in analysing rate-dependent effects, i.e. the positive force-frequency staircase effects, even if thermodynamic and mechanical instabilities would deserve a more careful inspection in that case.

These effects will be incorporated in a forthcoming contribution. In particular, our perspective is to couple the model presented here with accurate voltage plus calcium electrophysiological dynamics, i.e. Luo-Rudy or Beeler-Reuter models (Pullan et al., 2005). Moreover, an experimental fine-tuning of our model with stretch-activated channels (Seol et al., 2008) would introduce a further level of complexity, allowing for a full bidirectional electromechanical coupling. Therefore, novel simulationbased predictions of single myocyte contraction could be obtained both for physiological (Nishimura et al., 2008) and pathological conditions (Ward et al., 2008). We foresee the application of our model in describing the organization and remodelling of the internal myocyte structures during contraction (Kockskåmper et al., 2008) and the description of the diastolic calcium homeostasis as well, via a careful modelling of the interplay between the SR calcium re-uptake and the calcium extrusion systems (Louch et al., 2012). This allows our modelling approach to be extended and analysed for the failing myocardium conditions. Non-local constitutive laws could be adopted for mimicking microstructure cellular adaptation to the external substrate (Novak et al., 2004). The present model could be further adopted to answer the cell biomechanics challenges regarding the measure of the mechanical properties of living cells that react to the measurement tools. Because the model captures the effects of the external environment on the cellular structure, it could be used as a framework to design and interpret advanced experiments in this direction. Finally, we stress that our model could be employed as a building block for a multiscale cardiac model integrating cell, tissue and organ levels, with particular interest for the role of mechanoelectric feedback in the vulnerability to electric shocks (Li et al., 2008) and in tissue pinning phenomena associated with arrhythmias (Pumir et al., 2005; Cherubini et al., 2012).

Another limitation of the present work consists of the lack of a detailed description of cross-bridge dynamics (Rice et al., 2008). Although calcium velocities of the proposed model show satisfactory results, several features of contracting cardiomyocytes, like isometric force-length relationship, isotonic force-velocity relationships (Iribe et al., 2009) and stimulation frequency effects on calcium cycling (Louch et al., 2012), have not been tested. A following contribution will also take these aspects into account. We consider the present approach as a trade-off between sophisticated ionic models that do not consider a continuum mechanics description of the cell itself, and hyperelastic anisotropic models for cardiomyocytes not including interaction with precise calcium dynamics. In this 
perspective a direct experimental test bed for such a modelling is represented by cell culture studies (Parker et al., 2008; Zhang et al., 2008; Grosberg et al., 2011; McCain et al., 2012). On these bases, a consistent validation of the feedback of the proposed calcium-active contraction coupling could be obtained.

\section{Acknowledgement}

We are grateful to Sathya Srinivasan (University of Calgary) for providing confocal images of cardiomyocytes.

\section{Funding}

We also acknowledge the financial support by the European Research Council through the Advanced Grant Mathcard, Mathematical Modelling and Simulation of the Cardiovascular System, Project 227058 and the International Center for Relativistic Astrophysics Network, ICRANet.

\section{REFERENCES}

Berry, M. F., Engler, A. J., Woo, Y. J., Pirolli, T. J., Bish, L. T., Jayasankar, V., Morine, K. J., Gardner, T. J., Discher, D. E. \& Sweeney, H. L. (2006) Mesenchymal stem cell injection after myocardial infarction improves myocardial compliance. Am. J. Physiol. Heart. Circ. Physiol., 290, H2196-2203.

Bers, D. M. (2002) Cardiac excitation-contraction coupling. Nature, 415, 198-205.

Böl, M., Schmitz, A., Götz, N. \& Siebert, T. (2012) A three-dimensional chemo-mechanical continuum model for smooth muscle contraction. J. Mech. Behav. Biomed. Mater., 13(C), 215-229.

Cherubini, C., FilipPI, S. \& GizzI, A. (2012) Electroelastic unpinning of rotating vortices in biological excitable media. Phys. Rev. E, 85, 031915.

Cherubini, C., Filippi, S., Nardinocchi, P. \& Teresi, L. (2008) An electromechanical model of cardiac tissue: Constitutive issues and electrophysiological effects. Prog. Biophys. Mol. Biol., 97, 562-573.

Cherubini, C., Filippi, S., NArdinocchi, P. \& Teresi, L. (2009) Electromechanical modelling of cardiac tissue. Mechanosensitivity of the Heart Series: Mechanosensitivity in Cells and Tissues, vol. 3 (A. Kamkin \& I. Kiseleva eds). Berlin: Springer.

Delbridge, L. M. D. \& Roos, K. P. (1997) Optical methods to evaluate the contractile function of unloaded isolated cardiac myocytes. J. Mol. Cell Cardiol., 29, 11-25.

Deshpande, V. S., McMeeking, R. M. \& Evans, A. G. (2006) A bio-chemo-mechanical model for cell contractility. PNAS, 103, 14015-14020.

Deshpande, V. S., Mrksich, M., McMeeking, R. M. \& Evans, A. G. (2008) A bio-mechanical model for coupling cell contractility with focal adhesion formation. J. Mech. Phys. Solids, 56, 1484-1510.

Fabiato, A. (1989) Appraisal of the physiological relevance of two hypothesis for the mechanism of calcium release from the mammalian cardiac sarcoplasmic reticulum: calcium-induced release versus charge-coupled release. Mol. Cell. Biochem., 89, 135-140.

Geuzaine, C. \& Remacle, J. F. (2009) Gmsh: a three-dimensional finite element mesh generator with built-in preand post-processing facilities. Int. J. Numer. Meth. Engrg., 79, 1309-1331.

Goktepe, S., AвiLez, O. J. \& Kuhl, E. (2010) A generic approach towards finite growth with examples of athlete's heart, cardiac dilation, and cardiac wall thickening. J. Mech. Phys. Solids, 58, 1661-1680.

Goldbeter, A., Dupont, G. \& Berridge, M. J. (1990) Minimal model for signal-induced $\mathrm{Ca}^{2+}$ oscillations and for their frequency encoding through protein phosphorylation. Proc. Natl. Acad. Sci. USA, 87, 1461-1465.

Goldmann, W. H. (2012) Mechanotransduction in cells. Cell. Biol. Int., 36, 567-70.

Grosberg, A., Kuo, P. L., Guo, C. L., Geisse, N. A., Bray, M. A., Adams, W. J., Sheehy, S. P. \& Parker, K. K. (2011) Self-organization of muscle cell structure and function. PLoS Comp. Biol., 7, e1001088. 
Hatano, A., Okada, J., Washio, T., Hisada, T. \& Sugiura, S. (2011) A three-dimensional simulation model of cardiomyocyte integrating excitation-contraction coupling and metabolism. Biophys. J., 101, 2601-2610.

Holzapfel, G. A. (2000) Nonlinear Solid Mechanics. A Continuum Approach for Engineering. Chichester: John Wiley \& Sons.

Holzapfel, G. A. \& Ogden, R. W. (2009) Constitutive modelling of passive myocardium: a structurally based framework for material characterization. Philos. Trans. R. Soc. Lond. A, 367, 3445-3475.

Humphrey, J. D. (2001) Stress, strain, and mechanotransduction in cells. J. Biomech. Eng., 123, 638-641.

Huxley, A. F. (1957) Muscle structure and theories of contraction. Prog. Biophys. Chem., 7, 255-318.

Iribe, G., Helmes, M. \& KoHL, P. (2007) Force-length relations in isolated intact cardiomyocytes subjected to dynamic changes in mechanical load. Am. J. Physiol. Heart Circ. Physiol., 292, H1487-H1497.

Iribe, G., Ward, C. W., Camelliti, P., Bollensdorff, C., Mason, F., Burton, R. A. B., Garny, A., Morphew, M., Hoenger, A., Lederer, W. J. \& Kohl, P. (2009) Axial stretch of rat single ventricular cardiomyocytes causes an acute and transient increase in $\mathrm{Ca}^{2+}$ spark rate. Circ. Res., 104, 787-795.

Ishide, N., Urayama, T., Inoue, K., Komaru, T. \& Takishima, T. (1990) Propagation and collision characteristics of calcium waves in rat myocytes. Am. J. Physiol. Heart, 259, H940-H950.

Iyer, V., Mazhari, R. \& WinsLow, R. L. (2004) A computational model of the human left ventricular epicardial myocyte. Biophys. J., 87, 1507-1525.

Katsnelson, L. B., Sulman, T. B., Solovyova, E. \& Markhasin, V. S. (2009) Cooperative mechanisms of thin filament activation and their contribution to the myocardial contractile function: assessment in a mathematical model. Biophysics, 54, 39-46.

KeEner, J. \& Sneyd, J. (1998) Mathematical Physiology. New York: Springer.

Kockskåmper, J., von Lewinski, D., Khafaga, M., Elgner, A., Grimm, M., Eschenhagen, T., Gottlieb, P. A., SACHS, F. \& PiesKe, B. (2008) The slow force response to stretch in atrial and ventricular myocardium from human heart: functional relevance and subcellular mechanisms. Prog. Biophys. Mol. Biol., 97, 250-267.

LaAdhari, A., Quarteroni, A. \& Ruiz-Baier, R. (2012) An Eulerian finite element method for the simulation of cardiomyocyte active contraction, submitted.

LANDESBerg, A. \& Sideman, S.(1994) Mechanical regulation of cardiac muscle by coupling calcium kinetics with cross-bridge cycling: a dynamic model. Am. J. Physiol., 267, H779-H795.

Li, W., Gurev, V., McCulloch, A. D. \& Trayanova, N. A. (2008) The role of mechanoelectric feedback in vulnerability to electric shock. Prog. Biophys. Mol. Biol., 97, 461-478.

Li, J., PAtel, V. V. \& Radice, G. L. (2006) Dysregulation of cell adhesion proteins and cardiac arrhythmogenesis. Clin. Med. Res., 4, 42-52.

Louch, W. E., Stokke, M. K., Sjaastad, I., Christensen, G. \& Sejersted, O. M. (2008) No rest for the weary: diastolic calcium homeostasis in the normal and failing myocardium. Physiology, 27, 308-323.

Marshall, K. L. \& LumpKin, E. A. (2012) The molecular basis of mechanosensory transduction. Adv. Exp. Med. Biol., 739, 142-55.

McCain, M. L., Lee, H. L., Aratyn-Schaus, Y., Kléber, A. G. \& Parker, K. K. (2012) Cooperative coupling of cell-matrix and cell-cell adhesions in cardiac muscle. PNAS, 109, 9881-9886.

Moireau, P., Xiao, N., Astorino, M., Figueroa, C. A., Chapelle, D., Taylor, C. A. \& Gerbeau, J. F. (2012) External tissue support and fluid-structure simulation in blood flows. Biomech. Model. Mechanobiol., 11, 1-18.

NARdinocchi, P. \& Teresi, L. (2007) On the active response of soft living tissues. J. Elasticity, 88, 27-39.

Negroni, J. A. \& Lascano, E. C. (2008) Simulation of steady state and transient cardiac muscle response experiments with a Huxley-based contraction model. J. Mol. Cell. Cardiol., 45, 300-312.

Nobile, F., QuARTERoni, A. \& Ruiz-Baier, R. (2012) An active strain electromechanical model for cardiac tissue. Int. J. Numer. Meth. Biomed. Engrg., 28, 52-71.

Novak, I. L., Slepchenko, B. M., Mogilner, A. \& Loew, L. M. (2004) Cooperativity between cell contractility and adhesion. Phys. Rev. Lett., 93, 268109.

Nishimura, S., Seo, K., Nagasaki, M., Hosoya, Y., Yamashita, H., Fujita, H., Nagai, R. \& Sugiura, S. (2008) Responses of single-ventricular myocytesto dynamic axial stretching. Prog. Biophys. Mol. Biol., 97, 282-297. 
Okada, J., Sugiura, S., Nishimura, S. \& Hisada, T. (2005) Three-dimensional simulation of calcium waves and contraction in cardiomyocytes using the finite element method. Amer. J. Physiol. Cell Physiol., 288, C510-C522.

Parker, K. K., TAn, J., Chen, C. S. \& Tung, L. (2008) Myofibrillar architecture in engineered cardiac myocytes. Circ. Res., 103, 340-342.

Pullan, A. J., Buist, M. L. \& Cheng, L. K. (2005) Mathematically Modeling the Electrical Activity of the Heart: From Cell to Body Surface and Back. Singapore: World Scientific.

Pumir, A., Sinha, S., Sridhar, S., Argentina, M., Horning, M., Filippi, S., Cherubini, C., Luther, S. \& KRINSKY, V. (2010) Wave-train-induced termination of weakly anchored vortices in excitable media. Phys. Rev. E, 82, 010901(R).

Quarteroni, A. \& Valli, A. (1997) Numerical Approximation of Partial Differential Equations. Berlin: Springer.

Rice, J. J. \& DE Tombe, P. P. (2004) Approaches to modeling cross-bridges and calcium-dependent activation in cardiac muscle. Prog. Biophys. Mol. Biol., 85, 179-195.

Rice, J. J., Wang, F., Bers, D. M. \& DE Tombe, P. P. (2008) Approximate model of cooperative activation and cross-bridge cycling in cardiac muscle using ordinary differential equations. Biophys. J., 95, 2368-2390.

Ronan, W., Deshpande, V. S., McMeeking, R. M. \& McGarry, J. P. (2012) Numerical investigation of the active role of the actin cytoskeleton in the compression resistance of cells. J. Mech. Behav. Biomed. Mater., 14, 143-157.

Rossi, S., Ruiz-Baier, R., Pavarino, L. F. \& Quarteroni, A. (2012) Orthotropic active strain models for the numerical simulation of cardiac biomechanics. Int. J. Numer. Meth. Biomed. Engrg., 28, 761-788.

Ruiz-Baier, R., Ambrosi, D., Pezzuto, S., Rossi, S. \& Quarteroni, A. (2013) Activation models for the numerical simulation of cardiac electromechanical interactions. Computer Models in Biomechanics: From Nano to Macro (G. A. Holzapfel and E. Kuhl, eds). Heidelberg: Springer, pp. 189-201.

Seol, C. A., Kim, W. T., Ha, J. M., Choe, H., Jang, Y. J., Youm, J. B., Earm, Y. E. \& Leem, C. H. (2008) Stretch-activated currents in cardiomyocytes isolated from rabbit pulmonary veins. Prog. Biophys. Mol. Biol., 97, 217-231.

Sheehy, S. P., Grosberg, A., Parker, K. K. (2012) The contribution of cellular mechanotransduction to cardiomyocyte form and function Biomech. Model. Mechanobiol. 11, 1227-1239.

SNEYD, J., EDs. (2005) Tutorials in Mathematical Biosciences II: Mathematical Modeling of Calcium Dynamics and Signal Transduction. Berlin: Springer. ISBN 978-3-540-25439-3.

SRINIVASAN, S. (2011) A method to fix and permeabilize isolated adult mouse cardiomyocytes for immuno-staining and confocal imaging. Nat. Protoc. doi:10.1038/protex.2011.235.

Stålhand, J., Klarbring, A. \& Holzapfel, G. A. (2008) Smooth muscle contraction: mechanochemical formulation for homogeneous finite strain. Prog. Biophys. Mol. Biol., 96, 465-481.

StÅlhand, J., Klarbring, A. \& Holzapfel, G. A. (2011) A mechanochemical 3D continuum model for smooth muscle contraction under finite strains. J. Theor. Biol., 268, 120-130.

STERn, M. D. (1992) Theory of excitation-contraction coupling in cardiac muscle. Biophys. J., 63, 497-517.

Strobeck, J. E. \& Sonnenblick, E. H. (1986) Myocardial contractile properties and ventricular performance. The Heart and Cardiovascular System (Fozzard H. A. et al. eds). New York: Raven Press.

Subramanian, S., Viatchenko-Karpinski, S., Lukyanenko, V., Györk, S. \& Wiesner, T. F. (2001) Underlying mechanisms of symmetric calcium wave propagation in rat ventricular myocytes. Biophys. J., 80, 1-11.

TABer, L. A. (2001) Biomechanics of cardiovascular development. Annu. Rev. Biomed. Eng., 3, 1-25.

TAKAMATSU, T. \& WiER, W. G. (1990) Calcium waves in mammalian heart: quantification of origin, magnitude, waveform and velocity. FASEB, 4, 1519-1525.

TracQui, P. \& OHAYon, J. (2009) An integrated formulation of anisotropic force-calcium relations driving spatiotemporal contractions of cardiac myocytes. Philos. Trans. R. Soc. Lond. A, 367, 4887-4905.

Tracqui, P., Ohayon, J. \& Boudou, T. (2008) Theoretical analysis of the adaptive contractile behaviour of a single cardiomyocyte cultured on elastic substrates with varying stiffness. J. Theor. Biol., 255, 92-105.

Ward, M. L., Williams, I. A., Chu, Y., Cooper, P. J., Ju, Y. K. \& Allen, D. G. (2008) Stretch-activated channels in the heart: contributions to length-dependence and to cardiomyopathy. Prog. Biophys. Mol. Biol., 97, 232-249. 
Washio, T., Okada, J. Sugiura, S.\& Hisada, T. (2012) Approximation for cooperative interactions of a spatiallydetailed cardiac sarcomere model. Cell. Mol. Bioeng., 5, 113-126.

Wong, J., GöKtePE, S. \& KuHL, E. (2013) Computational modeling of chemo-electro-mechanical coupling: a novel implicit monolithic finite element approach, submitted.

ZaHALAK, G. I. (1981) A distribution-moment approximation for kinetic theories of muscular contraction. Math. Biosci., 55, 89-114.

Zhang, Y., Sekar, R. B., McCulloch, A. D. \& Tung, L. (2008) Cell cultures as models of cardiac mechanoelectric feedback. Prog. Biophys. Mol. Biol., 97, 367-382.

Zimmermann, B. \& Walz, B. (1997) Serotinin-induced intercellular calcium waves in salivary glands of the blowfly Calliphora erythrocephala. J. Physiol., 500, 17-28.

\section{Appendix. Linearized equations}

The derivatives of the usual and modified invariants of the Cauchy-Green deformation tensor $\mathbf{C}$ with respect to the deformation gradient $\mathbf{F}$ are

$$
\begin{aligned}
& \frac{\partial I_{1}}{\partial \mathbf{F}}=2 \mathbf{F}, \quad \frac{\partial I_{4, f}}{\partial \mathbf{F}}=2 \mathbf{F} \mathbf{f}_{0} \otimes \mathbf{f}_{0}, \quad \frac{\partial I_{1}^{\mathrm{E}}}{\partial \mathbf{F}}=2\left(1+\gamma_{\mathrm{f}}\right)^{2} \mathbf{F}-2 g\left(\gamma_{\mathrm{f}}\right) \mathbf{F} \mathbf{f}_{0} \otimes \mathbf{f}_{0}, \\
& \frac{\partial I_{4, f}^{\mathrm{E}}}{\partial \mathbf{F}}=2\left(1+\gamma_{\mathrm{f}}\right)^{-2} \mathbf{F} \mathbf{f}_{0} \otimes \mathbf{f}_{0} .
\end{aligned}
$$

In addition, we write the derivatives of the following quantities in the direction of a material displacement increment $\delta \mathbf{u}$ :

$$
\begin{aligned}
& D \mathbf{F}[\delta \mathbf{u}]=\nabla \delta \mathbf{u}, \quad D J[\delta \mathbf{u}]=J \mathbf{F}^{-\mathrm{T}} \nabla \delta \mathbf{u}, \quad D \mathbf{F}^{-\mathrm{T}}[\delta \mathbf{u}]=-\mathbf{F}^{-\mathrm{T}}(\nabla \delta \mathbf{u})^{\mathrm{T}} \mathbf{F}^{-\mathrm{T}}, \\
& D\left(\frac{\partial I_{1}}{\partial \mathbf{F}}\right)[\delta \mathbf{u}]=2 \nabla \delta \mathbf{u F}, \quad D\left(\frac{\partial I_{4, f}}{\partial \mathbf{F}}\right)[\delta \mathbf{u}]=2 \nabla \delta \mathbf{u} \mathbf{F} \mathbf{f}_{0} \otimes \mathbf{f}_{0}, \\
D \mathbf{P}[\delta \mathbf{u}]= & 4 b \psi_{1}^{\mathrm{E}}\left(1+\gamma_{\mathrm{f}}\right)\left[\left(1+\gamma_{\mathrm{f}}\right) \mathbf{F}: \nabla \delta \mathbf{u}+g\left(\gamma_{\mathrm{f}}\right) \mathbf{F} \mathbf{f}_{0} \cdot \nabla \delta \mathbf{u} \mathbf{f}_{0}\right] \mathbf{F}+2\left(1+\gamma_{\mathrm{f}}\right) \psi_{1}^{\mathrm{E}} \nabla \delta \mathbf{u} \\
& +\left\{4 b \psi_{1}^{\mathrm{E}} g\left(\gamma_{\mathrm{f}}\right)\left[\left(1+\gamma_{\mathrm{f}}\right) \mathbf{F}: \nabla \delta \mathbf{u}+g\left(\gamma_{\mathrm{f}}\right) \mathbf{F} \mathbf{f}_{0} \cdot \nabla \delta \mathbf{u f}_{0}\right]\right. \\
& \left.+\left(1+\gamma_{\mathrm{f}}\right)^{-4}\left[4 b_{f}\left(I_{4, f}^{\mathrm{E}}-1\right) \psi_{4, f}^{\mathrm{E}}+2 a_{f} \exp \left(b_{f}\left[I_{4, f}^{\mathrm{E}}-1\right]^{2}\right)\right] \mathbf{F} \mathbf{f}_{0} \cdot \nabla \delta \mathbf{u f}_{0}\right\} \mathbf{F} \mathbf{f}_{0} \otimes \mathbf{f}_{0} \\
& +2\left[g\left(\gamma_{\mathrm{f}}\right) \psi_{1}^{\mathrm{E}}+\left(1+\gamma_{\mathrm{f}}\right)^{-2} \psi_{4, f}^{\mathrm{E}}\right] \nabla \delta \mathbf{u f}_{0} \otimes \mathbf{f}_{0}-p\left(\mathbf{F}^{-\mathrm{T}}: \nabla \delta \mathbf{u}\right) \mathbf{F}^{-\mathrm{T}}+p J \mathbf{F}^{-\mathrm{T}}(\nabla \delta \mathbf{u})^{\mathrm{T}} \mathbf{F}^{-\mathrm{T}} .
\end{aligned}
$$

and the derivative of the stress tensor in the direction of a pressure increment $\delta p$ is $D \mathbf{P}[\delta p]=-\delta p J \mathbf{F}^{-\mathrm{T}}$. 